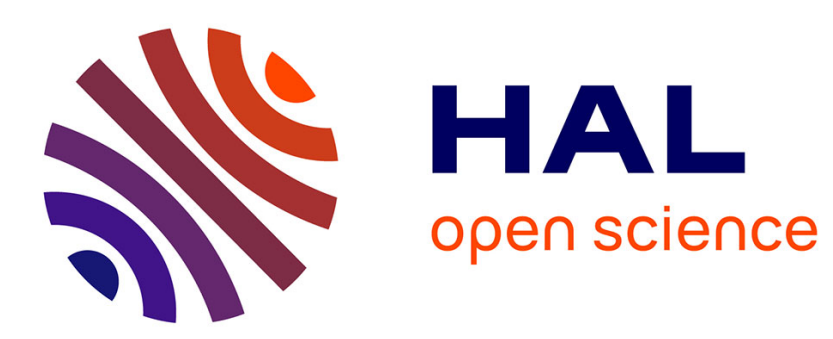

\title{
Theory of uniform-motion electromagnetic machines
}

\author{
G. Mourier
}

\section{To cite this version:}

G. Mourier. Theory of uniform-motion electromagnetic machines. Revue de Physique Appliquée, 1978, 13 (5), pp.252-270. 10.1051/rphysap:01978001305025200 . jpa-00244449

\section{HAL Id: jpa-00244449 https://hal.science/jpa-00244449}

Submitted on 1 Jan 1978

HAL is a multi-disciplinary open access archive for the deposit and dissemination of scientific research documents, whether they are published or not. The documents may come from teaching and research institutions in France or abroad, or from public or private research centers.
L'archive ouverte pluridisciplinaire HAL, est destinée au dépôt et à la diffusion de documents scientifiques de niveau recherche, publiés ou non, émanant des établissements d'enseignement et de recherche français ou étrangers, des laboratoires publics ou privés. 


\title{
THEORY OF UNIFORM-MOTION ELECTROMAGNETIC MACHINES
}

\author{
G. MOURIER \\ Electron Tube Division, THOMSON-C. S. F., Paris, France \\ (Reçu le 14 décembre 1977, accepté le 8 février 1978)
}

\begin{abstract}
Résumé. - Dans la première partie de cet article, on étudie les analogies entre les machines électromagnétiques classiques (moteurs, alternateurs, etc...) et les tubes électroniques de structure périodique, et on établit une théorie commune.

Dans la deuxième partie, cette théorie sert à définir de nouveaux types de machines tournantes pouvant fonctionner avec des courants occupant une bande de fréquence très large, ou à fréquence variable, ou indépendamment de la vitesse de rotation. On cite différentes applications de ces machines et on indique quelques-uns des problèmes de réalisation.
\end{abstract}

\begin{abstract}
In the first part of this paper, analogies between conventional rotating electromechanical machines (motors, alternators, etc...) and periodic-structure microwave electron tubes are made, and a common theory is elaborated.

In the second part, this theory is used to define new types of rotating machines that will operate with electrical currents having a very wide frequency spectrum, or at variable frequency, or independently of the rotational velocity. Different applications for these machines are envisaged and some problems involved in their use are mentioned.
\end{abstract}

\section{PART ONE : List of symbols.}

\begin{tabular}{|c|c|}
\hline$x, y, z, t$ & : Space-time coordinates (see later). \\
\hline $\mathbf{v}$ & : Velocity. \\
\hline$v$ & : Measure of volume. \\
\hline$e$ & : Electron charge. \\
\hline$m$ & : Electron mass. \\
\hline $\mathbf{E}, \mathbf{H}$ & : Electric, magnetic field. \\
\hline$\omega$ & : Angular frequency. \\
\hline$\rho$ & : Electron charge density. \\
\hline $\mathbf{J}$ & : Current density (convection). \\
\hline $\mathbf{J}_{\mathbf{t}}$ & $\begin{array}{l}\text { : Total current density (convection }+ \text { dis- } \\
\text { placement) }\end{array}$ \\
\hline$V$ & :Voltage \\
\hline$I$ & : Current. \\
\hline$\beta$ & : Propagation constant. \\
\hline$\beta_{\mathrm{r}}, \beta_{\mathrm{i}}$ & : Real and imaginary parts of $\beta$. \\
\hline$\mu$ & : Magnetic permeability. \\
\hline$\varepsilon$ & : Dielectric constant. \\
\hline $\mathbf{S}$ & : Poynting vector. \\
\hline$Z$ & : Impedance. \\
\hline$Y$ & : Admittance. \\
\hline $\mathbf{F}$ & : Force. \\
\hline $\mathbf{T}$ & : Maxwell's stress tensor. \\
\hline $\begin{array}{l}P_{\mathrm{m}}, P_{\mathrm{e}} \\
u\end{array}$ & $\begin{array}{l}\text { : Mechanical power, electrical power. } \\
\text { : Velocity of a moving circuit. }\end{array}$ \\
\hline$X$ & : Characteristic reactance. \\
\hline$\gamma$ & : Coefficient of exponential space variation. \\
\hline & : Constants. \\
\hline$K$ & : Surface current density. \\
\hline$K$ & : Pierce's coupling impedance. \\
\hline
\end{tabular}

$\begin{array}{ll}N & : \text { Number of windings per pole. } \\ P & : \text { Periodicity, or distance between poles. } \\ h & \text { : Width of a circuit }(z \text { direction). } \\ \sigma & : \text { Surface charge density. } \\ \kappa & : \text { Relativistic factor } \frac{1}{\sqrt{1-\frac{u^{2}}{c^{2}}}} \text { (generally } \\ & \\ & \quad \text { called } \gamma \text { in relativity theory). } \\ L_{\mathrm{n}}, \Lambda_{\mathrm{n}} & : \text { : Inductivities of components. } \\ C_{\mathrm{n}}, \Gamma_{\mathrm{n}} & : \text { Capacities of components. } \\ R & : \text { Resistances of components. } \\ \omega_{\mathrm{p}} & : \text { Plasma frequency of a gas of free charges. } \\ C & : \text { Pierce's coupling factor. } \\ A_{\mathrm{n}} & : \text { Coefficients in a MacLaurin expansion. } \\ g & : \text { Power gain per unit length in decibel } / \mathrm{m} .\end{array}$

1. Introduction. - There are certain essential resemblances between rotating machines, in particular multipole machines, and electron tubes in which an electronic cloud and a wave are propagated at closely similar velocities, for example, traveling-wave tubes and magnetrons. The resemblance is very close if there is considered a magnetron anode (active electromagnetic field components, in cylindrical coordinates $\left.r \varphi z, E_{r} E \varphi H_{z}\right)$ and the stator of an alternator from which the rotor has been removed (active components $\left.B_{r} B \varphi E_{z}\right)$. The field maps correspond by dual transformation. Those of the alternator are profoundly modified by the presence of the rotor, as in the case of 
high-current tubes. Note that the field maps of traveling-wave tubes have a different symmetry: the axis is longitudinal and not transversal relative to the motion but linear motors exist, too.

As concerns synchronism (velocity of the moving element and phase velocity close to each other), it will be observed that even asynchronous machines operate preferably close to synchronism, to within a few percent (which is the condition of good efficiency) and that synchronism is often not ensured in a more precise manner in traveling-wave tubes (TWT's). In either case, a certain modulation is produced in the moving circuit or the electronic cloud by the fixed circuit and this modulation, driven by the motion of the moving circuit, must remain in phase with the fields of the fixed circuit for a few periods until a new modulation is produced. The resemblances are therefore the following:

- Existence of a fixed part, having a periodic structure, and a moving part.

- Approximate synchronism between the moving part and the fields of the fixed part.

- Surface-wave fields of the magnetic type (also called transverse electric) in rotating machines and of the electric type (also termed transverse magnetic) in microwave tubes. One calls surface waves traveling electromagnetic fields with a high transverse variation (therefore very different from bulk waves), which exist only at the surface of physical circuits, generally having a periodic structure, and at phase velocities much lower than the velocity of light. The terminology magnetic or electric type is that of the waveguide theory and translates, in the present case, what has been mentioned above in respect to the essential field components.

A fourth resemblance can also be found :

- Function of these apparatus : conversion of electric energy into mechanical energy or vice versa. This is perfectly clear for the rotating machines and TWT's, which employ the kinetic energy of the beam, or linear accelerators.

In magnetrons, the phenomena are more complex since the electrons are accelerated by the dc field which prevails between the cathode and the anode (so that the source of voltage supplies the necessary energy), while being retarded by the high-frequency field (so that they give up energy to the latter).

It is obvious that, in generators, the moving part is always retarded by the fields of the fixed part whereas, in motors or accelerators, it is accelerated.

The following table can therefore be drawn up :

$\begin{array}{ccc}\text { Generating devices } & \text { Driving devices } \\ \text { «B type » machines } & \begin{array}{c}\text { Alternators } \\ \text { Hypersynchronous } \\ \text { generators } \\ \text { Magnetrons }\end{array} & \text { Motors } \\ \text { «E type » machines } & \begin{array}{c}\text { Traveling-wave tubes } \\ \text { Carcinotrons }\end{array} & \begin{array}{c}\text { Linear and most } \\ \text { cyclic } \\ \text { accelerators }\end{array}\end{array}$

It will also be observed that, in generators, the moving part often operates faster than the fields of the fixed part (and never slower), whereas the reverse is true of motors.

There are naturally large differences between these machines, to which reference will be made later. For example, the output energy is obtained from a single point in microwave tubes and from each pole of alternators; the moving part of machines is rigid whereas the electronic cloud deforms.

Before dealing with the differences, let us examine the resemblances in greater detail.

2. Electron beams considered as electrical circuits. One may wonder if it is theoretically possible to construct a TWT in which the electron beam would be replaced by a dielectric cylinder or any other ridig electric circuit traveling at the suitable velocity on the axis of a helix. Also, one can ask one-self what difference there is between these circuits and an electron beam.

To reply to these questions, it is convenient to consider the beam solely in its linear, or so called smallsignal operation. This is done in appendix $\mathrm{I}$.

In accordance with the analysis of the appendix, provided that we limit ourselves to linear operation and place ourselves in the frame of reference related to the electrons, the electron beam may be represented by an assembly of inductances and capacitances.

This simple démonstration does not take into account the magnetic field present in traveling-wave tubes, which renders the medium anisotropic and imparts new properties to it. But these properties exist in transformers having an anisotropic core (ferrite) and the electron beam may in any case be symbolized by equations that in no way differ from those of electric circuits in the linear approximation.

3. Interaction between a fixed circuit and a moving circuit - General equations. - 3.1 Assumptions. This interaction may be treated in a relatively simple manner in limiting ourselves to the most common forms of circuits and in idealizing them in the following manner :

3.1.1 The only systems considered are those of uniform or periodic structure in one direction and moving with respect to each other at uniform velocity.

3.1.2 For the sake of simplicity, the circular systems (rotating machines, magnetrons) are laid out along a direction $x$, the direction of the former axis still being called $\mathrm{O} z$.

Likewise, the cylindrical systems (TWT's) are replaced by bidimensional Cartesian systems in which $x$ is the direction of propagation, and the direction $\varphi$ becomes $z$. The systems are assumed to be infinite in the direction $z$.

3.1.3 Only machines having a very large number of 
poles per cycle, thus very small periodicity, are considered. The shape of the poles is idealized, as it will be seen.

The systems considered (Figs. 1 and 2) are therefore

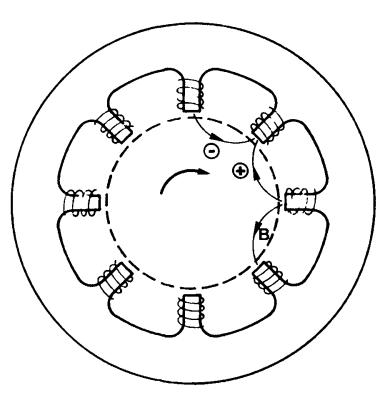

ALTERNATOR

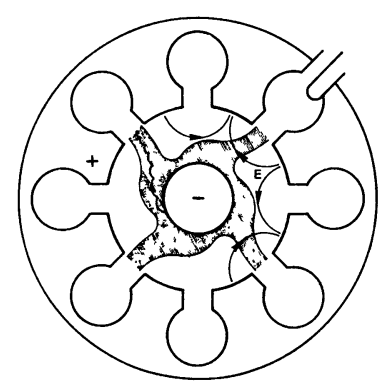

MAGNETRON
FiG. 1. - Diagrams showing some analogies between an alternator and a magnetron.

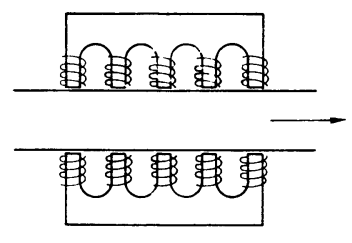

LINEAR MOTOR

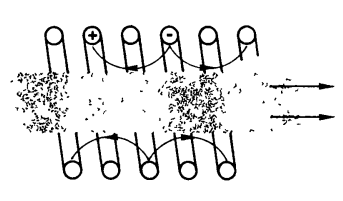

TRAVELING-WAVE TUBE
FIG. 2. - A linear motor and a travelling wave tube.

reduced to sections of substances characterized by the constants $\varepsilon, \mu$ and $\sigma$, which are traversed or not traversed in the direction $z$ (rotating machines) by conductors having a periodic structure (See Fig. 3). These

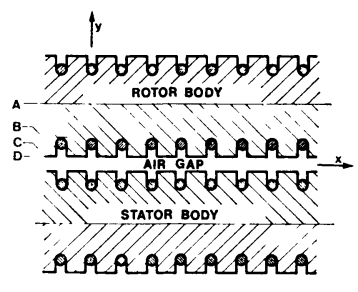

LINEAR MACHINE

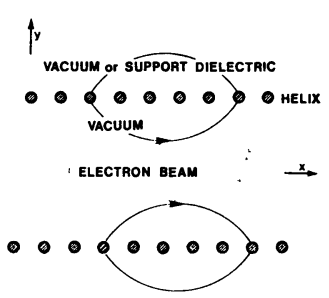

A TWO-DIMENSIONAL TWT
FIG. 3. - Analytic diagrams of a linear machine and a travelling wave tube.

systems are completely homogeneous in the direction $z$, necessarily non-homogeneous in the direction $y$, but formed of stratified homogeneous regions. In the direction $x$, the hypothesis of small periodicity, chosen for the sake of analytical simplification, will permit passing, at the limit, to homogeneous systems.

Each field component $A$, each current or voltage, is then assumed to vary as

$$
A=A_{0}(y) \mathrm{e}^{j(\omega t-\beta x)} .
$$

It is always possible to assume that $\omega$ and $\beta$ are known real quantities since the components may be subjected to Fourier's analysis. Complex values may also be used, which then describe damping or starting of oscillations, attenuations or amplifications.

The function $A_{0}$ may be defined throughout while having discontinuities, or be defined solely for discrete values of $y$ (voltages) or be composed of Dirac functions (current densities).

3.2 MAXWELL's EQUATIONS. - A first group of Maxwell's equations is written

$$
\begin{array}{ll}
\text { Magnetic system } & \text { Electric system } \\
j \omega \mu H_{x}=-\frac{\partial E_{z}}{\partial y} & j \omega \varepsilon E_{x}=\frac{\partial H_{z}}{\partial y} \\
j \omega \mu H_{y}=\frac{\partial E_{z}}{\partial x} & j \omega \varepsilon E_{y}==\frac{\partial H_{z}}{\partial x}
\end{array}
$$

where $\mu$ and $\varepsilon$ may be complex (conductivity).

The derivation with respect to $y$ is not possible throughout; the $x$-derivation transforms the equations (3), if (1) is taken into account, into :

$$
\omega \mu H_{y}=-\beta E_{z} \quad \omega \varepsilon E_{y}=\beta H_{z}
$$

The result is that the components parallel to the stratification surfaces contain all the information concerning each value of $y$, and the normal components $H_{y}$ and $E_{y}$ may be deduced if $\omega$ and $\beta$ are known. (This is well known, theorem in e. m. theory.)

The state of a surface $\Sigma$ of ordinate $y$ is thus completely determined if $\omega, \beta$ and the value of the independant components :

$$
H_{x} E_{z} \quad E_{x} H_{z}
$$

are given for a particular value of $x$.

The study of the complete system then, consists in relating these couples of values in different planes, on one hand, to each other and, eventually, to the voltages and currents in the conductors [3-7].

Before establishing these transformations, a few additional properties of the independent components may be established.

3.3 TransVerse POWER FluX, IMPEDANCE, SURFACE ADMITTANCE. - The word transverse is here understood to be with respect to the motion of the machine. Let us write the expression of the Poynting vector in the direction $y$ :

$$
S_{y}=E_{z} H_{x} \quad S_{y}=-E_{x} H_{z} .
$$

In complex notation :

$\bar{S}_{y}+j S_{j y}=\frac{1}{2} E_{z} H_{x}^{*} \quad \bar{S}_{y}+j S_{j y}=-\frac{1}{2} E_{x}^{*} H_{z}$

If the electric field opposites are made to perform the function of voltages, it is normal to take as currents in the direction $+y$ the quantities :

$$
-H_{x} \quad H_{z}
$$

and the impedances and admittances in the direction $+y$ are then defined : 


$$
\begin{array}{ll}
Z_{+y}=\frac{E_{z}}{H_{x}} & Z_{+y}=-\frac{E_{x}}{H_{z}} \\
Y_{+y}=\frac{H_{x}}{E_{z}} & Y_{+y}=-\frac{H_{z}}{E_{x}} .
\end{array}
$$

3.4 MeChANICAL FORCES. - The forces of electrical origin per unit volume may be evaluated by the formulas

$$
\frac{\mathrm{d} \mathbf{F}}{\mathrm{d} v}=\rho \mathbf{E}+\mathbf{J} \wedge \mathbf{B}
$$

or by employing the expression :

$$
\frac{\mathrm{d} F_{i}}{\mathrm{~d} v}=\frac{\partial}{\partial x_{j}} T_{i j}
$$

where $T$ is Maxwell's stress tensor. The most interesting forces are those applied in the direction $x$ to the surfaces perpendicular to $y$ because they tend to modify the velocity of the moving circuit. Further, only the derivation with respect to $y$ gives a non-null mean value since $d / d z=0$ and the magnitudes are alternating in the direction $x$.

It is sufficient to consider the equation :

$$
\frac{\mathrm{d} F_{x}}{\mathrm{~d} v}=\frac{\partial}{\partial y} T_{x y}
$$

The force per unit surface $f_{x}$ will be considered :

$$
\frac{\mathrm{d} f_{x}}{\mathrm{~d} y}=\frac{\partial}{\partial y} T_{x y} .
$$

For non-relativistic systems, this is reduced to :

$$
\frac{\partial f_{x}}{\partial y}=\frac{\partial}{\partial y} \mu H_{x} H_{y} \quad \frac{\partial f_{x}}{\partial y}=\frac{\partial}{\partial y} \varepsilon E_{x} E_{y} .
$$

The force applied to the moving-system is to be found by integration :

$$
f_{x}=\int_{\mathrm{D}}^{\mathrm{M}} \frac{\partial T_{x y}}{\partial y} \mathrm{~d} y=T_{x y / \mathrm{M}}-T_{x y / \mathbf{D}} .
$$

The point $\mathrm{M}$ is taken far enough away so that $T_{x y}$ vanishes. We then have for the force applied per unit to the moving part, simply :

$$
f_{x}=-\mu H_{x} H_{y} \quad f_{x}=-\varepsilon E_{x} E_{y} .
$$

In complex notation

$\bar{f}_{x}+j f_{j x}=-\frac{\mu}{2} H_{x} H_{y}^{*} \quad \bar{f}_{x}+j f_{j x}=-\frac{\varepsilon}{2} E_{x} E_{y}^{*}$.

Utilizing the relations (4) :

$$
f_{x}=H_{x} \frac{\beta}{\omega} E_{z} \quad f_{x}=-E_{x} \frac{\beta u}{\omega} H_{z} .
$$

There is thus a simple relation between $f_{x}$ and $S_{y}$ :

$$
f_{x}=\frac{\beta}{\omega} S_{y} .
$$

We must however realize that equation (4) is only valid in complex notation, unless $\beta$ and $\omega$ are real (which is the case of rotating machines and magnetrons in the operating state). Assuming that the moving part is driven at a velocity $u$ in the direction $x$, we can calculate the mechanical power $P_{\mathrm{m}}$ imparted to it. We have per unit surface :

$$
\frac{\mathrm{d}^{2} P_{\mathrm{m}}}{\mathrm{d} x \mathrm{~d} z}=u f_{x}=\frac{\beta u}{\omega} S_{y}=\frac{\beta u}{\omega} \frac{\mathrm{d}^{2} P_{\mathrm{e}}}{\mathrm{d} x \mathrm{~d} z} .
$$

This relation, which is solely valid in respect to traveling or rotating fields, permits in certain cases the calculation of the efficiency of a machine as a function of the slip. It is in particular necessary for this that the source of electrical energy used be connected solely to the stator or to the rotor :

$$
\rho=\frac{\beta u}{\omega} .
$$

For a generator, with the corresponding restrictions concerning the efficiency of the circuit, one has :

$$
\rho=\frac{\omega}{\beta u} .
$$

Note that $\omega / \beta$ is the phase velocity of the wave. We deduce the following consequences :

a) - In general, the closer to synchronism are circular machines, the higher their efficiency is (this does not apply to open electron tubes, in which the beam retains the kinetic energy at the output).

$\beta)$ - Motors, accelerators, etc., operate with a velocity of the moving part lower than the phase velocity $(\rho<1)$. The reverse is true in respect to generators and amplifiers.

Note moreover that $S_{y}$ and $f_{x}$ are products of alternating quantities that have a mean value (active power) but also an alternating part, which is the source of possible vibrations.

If $\omega$ or $\beta$ are complex, we obtain for the mean values with respect to time :

$$
\begin{aligned}
& \bar{f}_{x}=\frac{1}{4}\left[\frac{\beta^{*}}{\omega^{*}} H_{x} E_{z}^{*}+\frac{\beta}{\omega} H_{x}^{*} E_{z}\right] \\
& \bar{f}_{x}=-\frac{1}{4}\left[E_{x} \frac{\beta^{*}}{\omega^{*}} H_{z}^{*}+E_{x}^{*} \frac{\beta}{\omega} H_{z}\right]
\end{aligned}
$$

hence :

$$
\bar{f}_{x}=\bar{S}_{y}\left[\operatorname{Re} \frac{\beta}{\omega}\right]-S_{j y}\left[\operatorname{Im} \frac{\beta}{\omega}\right]
$$

Inversely, we may relate the mean $\bar{S}$ of the Poynting vector to the complex expression for $f_{x}$ :

$$
\bar{S}_{y}=\bar{f}_{x}\left[\operatorname{Re} \frac{\omega}{\beta}\right]+f_{j y}\left[\operatorname{Im} \frac{\omega}{\beta}\right] .
$$

These expressions are useful for calculating the efficiency of the machines in taking into account the 
Joule losses, or the amplification of a wave, which is expressed by a complex value of $\beta$.

4. Analytical study of the elements of the machines. - 4.1 TRANSFORMATION OF THE FIELDS IN A HOMOGENEOUS MEDIUM. - One desires, for example, to relate the fields between the two planar surfaces passing through the points A and B of figure 3 .

We complete the Maxwell equations with the second group which reduces to :

$$
+j \omega \varepsilon E_{z}=\frac{\partial H_{y}}{\partial x}-\frac{\partial H_{x}}{\partial y} \quad-j \omega \mu H_{z}=\frac{\partial E_{y}}{\partial x}-\frac{\partial E_{x}}{\partial y}
$$

combining with (2) and (4) we obtain :

$$
\frac{\partial^{2} E_{z}}{\partial y^{2}}=\gamma^{2} E_{z} \quad \frac{\partial^{2} H_{z}}{\partial y^{2}}=\gamma^{2} H_{z}
$$

in which we have set :

$$
\gamma^{2}=\beta^{2}-\omega^{2} \varepsilon \mu .
$$

According to the preceding paragraph, the second term is very often negligible since we generally have :

$$
u^{2} \ll \frac{1}{\varepsilon \mu} .
$$

We shall choose the root such that :

$$
\operatorname{Re} \gamma>0 \text {. }
$$

From there, it is easy to establish general expressions for the $y$-variations of the fields, and to calculate any field quantity as a function of two particular independant values. Of particular interest are the linear relations between four quantities considered as two sets, corresponding each two a given point in space, of two independant field quantities.

They can be expressed in different matrix forms exactly like the current and voltages of a 2 port network. (See appendix 2). For instance, one defines a dyadic $E_{z}, H_{x}$ for each value of $y$ and relate these dyadics by a matrix relation :

$$
\left.\left.H_{x}\right]_{y+h}=\left[\begin{array}{ll}
\cosh \gamma h & -j X \sinh \gamma h \\
+j \frac{\sinh \gamma y}{j X} & \cosh \gamma h
\end{array}\right]_{x}\right]_{y}
$$

where :

$$
X=\frac{\omega \mu}{\gamma} .
$$

Dual relations exist for the electric case.

In short, the medium is treated as a transverse transmission line with imaginary characteristic impedance and propagation constant.

4.2 Network voltages AND CURRENTS. - It is now desired to couple the fields in the planes $\mathrm{B}$ and $\mathrm{C}$ of figure 3. We will assume that there are many conductors of very small diameter per cycle counted in the direction $x$, so that these conductors may be replaced by an infinitely thin layer of currents directed in the direction $z$ and varying in accordance with equation (1) (we should very much like to achieve this without excessive Joule losses and mechanical complications). Let $K$ be the intensity per unit length measured in the direction $x$. The equations of continuity are :

$$
H_{x^{+}}=H_{x^{-}}=K \text {. }
$$

We designate by $H_{x}$ the value of $H_{x}$ for a $y$ very slightly greater algebraically than that of $K$, that is to say, for example, at $\mathrm{B}, H_{x^{-}}$being then considered at C. Further, with the same convention :

$$
E_{z^{+}}=E_{z^{-}}=E
$$

and, because of (4) :

$$
H_{y^{+}}=H_{y^{-}} .
$$

The spatial period of the winding was termed $p$. If the winding has $N$ wires per period, through which a current $I$ travels we have :

$$
K=\frac{N}{p} I .
$$

If, furthermore, the length of each wire in the direction $z$ is $h$, and if the return occurs, as in the figure 3 , in a region where the field is negligible, the voltage developed at the terminals of the winding is :

$$
V=-N E h \text {. }
$$

The sign could be chosen arbitrarily. We shall explain our choice later.

The equations (31) to (34) correspond to the magnetic case. An analogous transformation exists in the electrical case for a layer of charges :

$$
\begin{aligned}
& E_{y^{+}}=E_{y^{-}}=\frac{\sigma}{\varepsilon_{0}} \\
& H_{z^{+}}=H_{z^{-}}=\frac{\omega}{\beta} \sigma \\
& E_{x^{+}}=E_{x^{-}}=E .
\end{aligned}
$$

4.3 TRANSFORMATION OF THE FIELDS BETWEEN THE FIXED PART AND THE MOVING PART. - In electronic systems, these two parts are separated by an infinitely thin ideal surface. In electromechanical systems, there is always an airgap, which may be taken care of by the equations of appendix II in putting $\mu=\mu_{0}$. Further, it will be necessary to couple the fields in the materials, for example magnetic materials to the fields in the vacuum. Electromagnetic theory shows that the tangential components of the electric field and the magnetic field are continuous on the surface of separation.

But the equations were up till now written in a system related to the physical elements and they must be transformed in the fixed system if the moving part is concerned. 
Considering figure 3, it is desired to transform at D the components evaluated in the moving system, to which we shall affix the sign $\left(^{\prime}\right)$, into those of the fixed system. This operation is carried out in space by means of Lorentz's transformation which here reduces to the following expressions :

$$
\begin{array}{ll}
H_{x}=H_{x}^{\prime} & E_{x}=E_{x}^{\prime} \\
H_{y}=\kappa\left[H_{y}^{\prime}-u \varepsilon_{0} E_{z}^{\prime}\right] & E_{y}=\kappa\left[E_{y}^{\prime}+u \mu_{0} H_{z}^{\prime}\right] \\
E_{z}=\kappa\left[E_{z}^{\prime}-u \mu_{0} H_{y}^{\prime}\right] & H_{z}=\kappa\left[H_{z}^{\prime}+u \varepsilon_{0} E_{y}^{\prime}\right]
\end{array}
$$

where $u$ is the velocity of the moving system and :

$$
\kappa=\frac{1}{\sqrt{1-u^{2} / c^{2}}} .
$$

We have moreover

$$
\begin{aligned}
& \gamma^{\prime}=\gamma, \\
& \beta^{\prime}=\kappa\left(\beta-\omega u / c^{2}\right), \\
& \omega^{\prime}=\kappa(\omega-\beta u) .
\end{aligned}
$$

Combining equations (4) and (39), one obtains :

$$
\begin{array}{ll}
E_{z}=\kappa E_{z}^{\prime}\left[1+\frac{u \beta^{\prime}}{\omega^{\prime}}\right] & H_{z}=\kappa H_{z}^{\prime}\left[1+\frac{u \beta^{\prime}}{\omega^{\prime}}\right] \\
\frac{E_{z}}{\omega}=\frac{E_{z}^{\prime}}{\omega^{\prime}} & \frac{H_{z}}{\omega}=\frac{H_{z}^{\prime}}{\omega^{\prime}} .
\end{array}
$$

The equations (37) and (45) are sufficient to express the transformation of the fields within the framework of the hypotheses made, completed by the space-time equations (41) to (43).

Except in the case of accelerators or very high-voltage electron tubes, we may be satisfied with non-relativistic equations $\left(c^{2} \rightarrow \infty\right)$ :

$$
\begin{aligned}
& \gamma^{\prime}=\gamma \\
& \beta^{\prime}=\beta \\
& \omega^{\prime}=\omega-\beta u
\end{aligned}
$$

the equations (37) and (45) being unchanged. These equations belong therefore to the Galilean transformation.

4.4 THE IMPEDANCE OF UNIFORM SYSTEMS IN UNIFORM MOTION. - If the moving part is not connected to a source of energy, it may be characterized by its impedance or admittance, defined by the equations (6). According to the equations (32) and (33), they must obey, in the moving system, the general laws of electrical circuits. For example, if the resistive losses are negligible, they may be put in Foster's canonic forms :

$$
\begin{aligned}
& Z^{\prime}=j\left[L_{0}^{\prime} \omega^{\prime}-\frac{1}{C_{0}^{\prime} \omega^{\prime}}\right]+\sum_{n} \frac{1}{j\left[C_{n}^{\prime} \omega^{\prime}-\frac{1}{L_{n}^{\prime} \omega^{\prime}}\right]}, \\
& Y^{\prime \prime}=\left[j \Gamma_{0}^{\prime} \omega-\frac{1}{\Lambda_{0}^{\prime} \omega^{\prime}}\right]+\sum_{n} \frac{1}{j\left[\Lambda_{n}^{\prime} \omega^{\prime}-\frac{1}{\Gamma_{n}^{\prime} \omega^{\prime}}\right]} .
\end{aligned}
$$

According to equations (37) and (45); we have :

$$
\frac{Z^{\prime}}{\omega^{\prime}}=\frac{Z}{\omega} \quad \frac{Y^{\prime}}{\omega^{\prime}}=\frac{Y}{\omega} .
$$

In combining (44) or (45) with one of the equations (47) ; depending on whether it concerns a system of magnetic type or electrical type, we have the most general forms that the impedances and admittances of the systems in motion may have.

Simple examples show that these forms correspond to radically new properties.

If we interconnect the windings of a rotor, the impedance $Z^{\prime}$ will always be inductive. We then have :

$$
Z^{\prime}=j L_{0}^{\prime} \omega^{\prime}
$$

hence

$$
Z=j L_{0}^{\prime} \omega
$$

which gives us nothing new. But, if on the other hand, we manage to introduce a capacitive element, we shall have for example :

$$
Z=j\left[L_{0}^{\prime} \omega-\frac{\omega}{C_{0}^{\prime}(\omega-\beta u)^{2}}\right] .
$$

We can thus have a reactance which decreases as a function of the frequency. Such an impedance, connected in series with a resistance, would result in complex values of $\omega$ or $\beta$ which represent instabilities or an amplification, in particular when $\omega^{\prime}=\omega-\beta u$ is close to 0 , that is to say around synchronous operation.

The dual phenomenon occurs in electronic tubes; the equations (46) show that if the moving medium is purely capacitive (dielectric) it brings no new property to the fixed system. If, on the other hand, we manage to introduce therein an inductive element, or an element of similar expression, the system may oscillate or amplify if the value of $u$ is well chosen. It has been seen in paragraph 2 that the electronic beam has indeed this property.

A resistance could also be placed in series with the inductance, which will give :

$$
Z=j L_{0}^{\prime} \omega+R \frac{\omega}{\omega^{\prime}} .
$$

This is an unusual expression since the real part has a real pole. In this case, the moving part, with its mutually coupled inductive component and its resistive component, resembles a rotor of an asynchronous machine.

The consequences of this will be developed in Part Two.

4.5 TRANSFORMATIONS EFFECTED BY SLIDING CONTACTS AND COMMUTATORS. - Sliding contacts employing rings and brushes do not introduce a modification in the currents and voltages: They transform in an identical manner the currents and voltages of the 
moving system to the fixed frame of reference. There only results, in particular, that the frequency remains that of the moving system, $\omega^{\prime}=\omega-\beta u$. The reason that the frequency does not obey Galileo's transformation $\left(43^{\prime}\right)$ in this case, is that there is on the rings no variation in the currents and voltages, which then do not follow equation (1), as the electrical quantities in the stator and rotor do.

Let us now consider a commutator system such that there corresponds to each winding or pole a commutator sector on the rotor with as many brushes fixed on the rotor. Instantaneously, each brush is in contact with either one or two sectors and vice versa, but both cases are practically equivalent as long as the hypothesis of many conductors per cycle is satisfied.

Each sector of the commutator is referred to by a value of $x^{\prime}$, and each brush by a value of $x$. At the moment of the contact, which occurs at a given $x$ in the fixed frame of reference, we have simply :

$$
\begin{aligned}
& V_{\text {brush }}=V_{\text {sector }}, \\
& I_{\text {brush }}=I_{\text {sector }} .
\end{aligned}
$$

The values on the sectors are the expressions contained in equations (32) and (33).

Equations (50) and (51) are satisfied on each brush at every instant, the sectors moving with respect to time. The frequency of the currents in the brushes is therefore not the same as in the sectors of the commutator. But, as the phase on the latter corresponds and is distributed, as in the poles, by the equations (1), the Galilean transformation applies, and in particular equation $\left(43^{\prime}\right)$.

Briefly, the commutator is, within the acceptable approximations, an element which conserves the currents and the voltages of the elements which are momentarily at the same abscissa, and which transforms the frequency of the rotor into the frequency of the fixed system, that is, the stator.

This is so, irrespective of the velocity $u$ and the frequency, and it is precisely what imparts to machines having a commutator their well-known versatility.

4.6 COUPLING TO A DELAY LINE. - In travelingwave tubes [1], the charges of the beam induce image charges in the conductors of the circuit, which may be represented as a delay line or a filter. If the phase velocity is roughly constant, a model of a low-pass filter may be employed (see Fig. 4).

In the case of rotating machines, there would be an analogous situation if the windings of a stator were

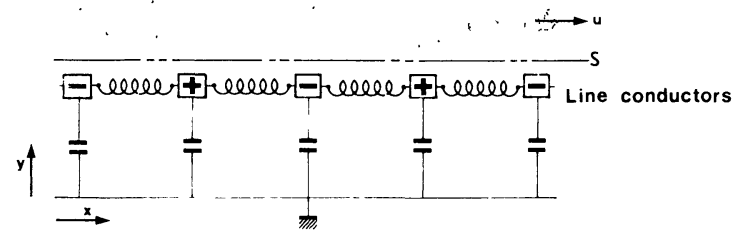

FIG. 4. - Electric circuit equivalent to a section of a travelling wave tube. connected with capacitances so as to form a delay line. Equations (32) and (33), completed by the circuit equations, enable this case to be treated, whereas equations (34), (35), (36) correspond to the case of microwave tubes.

There is, however, a more rapid method of treatment, which is valid in the case where the fixed part is connected as a filter. Let us consider on figure 4 an ideal surface $S$ of ordinate $Y_{\mathrm{s}}$, in the vicinity of the conductors. The moving part will be disposed above $S$.

As the electromagnetic fields of the circuit extend beyond $S$, the propagation in the circuit depends on the fields in this region. In order to characterize them, it is sufficient, in supposing that $\omega$ and $\beta$ are known, to specify at $Y_{\mathrm{s}}$ the components of the fields parallel to $S$, namely.

$$
E_{y}, H_{x} \quad H_{y}, E_{x} .
$$

The amplitude being undetermined in a linear system as long as the sources external to this system are not specified, it is sufficient to consider the ratio between the independent field components mentioned above. The system above the plane $S$ may therefore be characterized by the impedances or admittances that it produces in that plane :

$Z_{\mathrm{s}}=Z_{+y}=\frac{E_{\mathrm{z}}\left(Y_{\mathrm{s}}\right)}{H_{x}\left(Y_{\mathrm{s}}\right)} \quad Z_{\mathrm{s}}=Z_{+y}=-\frac{E_{x}\left(Y_{\mathrm{s}}\right)}{H_{z}\left(Y_{\mathrm{s}}\right)}$

As all the quantities obey equation (1), this impedance is independent of $x$ and $t$.

It must be equated with the impedance evaluated for the part below $S$, which contains the circuit :

$$
Z_{\mathrm{s}}=\left.\frac{E_{z}}{H_{x}}\right|_{y_{\mathrm{s}}, \text { circuit }} \quad Z_{\mathrm{s}}=-\left.\frac{E_{x}}{H_{z}}\right|_{y_{\mathrm{s}}, \text { circuit }} .
$$

In fact, we set :

$$
Z_{\mathrm{c}}=-\left.\frac{E_{z}}{H_{x}}\right|_{y_{\mathrm{s}}, \text { circuit }} \quad Z_{\mathrm{c}}=+\left.\frac{E_{x}}{H_{z}}\right|_{y_{\mathrm{s}}, \text { circuit }} .
$$

The change of sign indicates that we shall consider as positive the currents in the direction $-y$, that is to say those entering the circuit, which is thus considered as a load.

Noting that $Z_{\mathrm{s}}$ and $Z_{\mathrm{c}}$ are functions of $\omega$ and of $\beta$ (and of the geometric dimensions and the electric characteristics of the materials and possibly $u$ ), the complete equation of the circuit thus formed will be written :

$$
Z_{\mathrm{s}}(\omega, \beta)+Z_{\mathrm{c}}(\omega, \beta)=0 .
$$

This generally transcendental equation permits calculating $\omega$ as a function of $\beta$ or vice versa, and obtaining the value of the amplification or the real or complex values of $\omega$, depending on whether the system is stable or unstable. There as several approximate methods for solving such transcendental equations. 
5. Circuit equations of the machines. - We have expressed in sections 4.1 to 4.5 the properties of most of the homogeneous parts of the devices in analytical forms which, properly combined, will describe the behavior of the whole. We have however left out that which concerns the ordinary technique of circuits : interconnection of the windings, description of the excitation, etc.

Starting with the diagram of figure 3 , we shall completely describe a machine within the framework of the hypotheses made, in considering for each critical value of $y$ the components $E$ and $H$, the indices of which will be omitted in the different media, and in effecting the transformations of sections 1 to 5 .

We shall effect this calculation in simplifying as far as possible the algebraic expressions, to bring out the physical phenomena in the simplest manner.

Let us suppose that the magnetic circuit of the rotor is rather extensive in the direction $y$ and that the magnetic permeability is high, so that the fields are very small at $\mathrm{A}$. Let us place the origin of the $y_{\mathrm{s}}^{\prime}$ in the plane of the conductors between the very close points $B$ and $C$. We then have :

$$
\cosh \left(\gamma y_{\mathrm{A}}\right) \simeq \sinh \left(\gamma y_{\mathrm{A}}\right) \simeq \mathrm{e}^{\gamma Y \mathrm{~A}} / 2 .
$$

Thus, if $E\left(y_{\mathrm{A}}\right)$ is null, we have according to $(29 a)$ :

$$
E_{\mathrm{B}}=\frac{j \omega \mu H_{\mathrm{B}}}{\gamma} \text {. }
$$

To pass to the point $\mathrm{C}$, the transformations of equations (29) and (30) are employed :

$$
\begin{aligned}
& E_{\mathrm{C}}=E_{\mathrm{B}} \\
& H_{\mathrm{C}}=H_{\mathrm{B}}+K .
\end{aligned}
$$

To pass to the point $\mathrm{D}$, the transformations of the equations appendix II are employed, with :

$$
y_{\mathrm{D}}=-d
$$

we obtain, according to the developments of section 4.1

$$
\left.\begin{array}{c}
E_{\mathrm{D}}=E_{\mathrm{C}} \cosh (\gamma d)+\frac{j \omega \mu}{\gamma} H_{\mathrm{C}} \sinh (\gamma d) \\
-\frac{j \omega \mu}{\gamma} H_{\mathrm{D}}=-E_{\mathrm{C}} \gamma \sinh (\gamma d)-\frac{j \omega \mu}{\gamma} H_{\mathrm{C}} \cosh (\gamma d)
\end{array}\right\}
$$

Combining with the equations (58) and (59) :

$$
\left.\begin{array}{l}
E_{\mathrm{D}}=E_{\mathrm{B}} \mathrm{e}^{\gamma d}+\frac{j \omega \mu}{\gamma} K \sinh (\gamma d) \\
H_{\mathrm{D}}=\frac{\gamma}{j \omega \mu} E_{\mathrm{B}} \mathrm{e}^{\gamma d}+K \cosh (\gamma d)
\end{array}\right\} .
$$

Note that $E_{\mathrm{B}}$ and $K$ are quantities which permit the calculation of the voltages and the currents of the conductors by means of the equations (32) and (33).

These equations therefore relate the voltage and the current of the moving circuit to the electric and magnetic fields in the surface of the frame adjacent to the air gap. They describe the electrical behaviour of a circuit, rotor or stator.

To continue the calculation, we could go on effecting the transformations from point to point toward the negative $y_{s}^{\prime}$. But it is simpler to utilize the similarity of the structures of the stator and rotor. Indeed, the stator obeys a set of equations analagous to (62), but extending toward the negative $y_{\mathrm{s}}^{\prime}$. To change the sign of $y$, the axes must be rotated about the $z$ axis (so as to avoid modifying the expressions in rotational and derivatives), which requires changing the sign of $H_{x}$ everywhere.

We then have for the fields of the stator at D :

$$
\left.\begin{array}{l}
E_{\mathrm{Ds}}=E_{\mathrm{s}} \mathrm{e}^{\gamma d_{\mathrm{s}}}+\frac{j \omega \mu}{\gamma} K_{\mathrm{s}} \sinh \left(\gamma d_{\mathrm{s}}\right) \\
H_{\mathrm{Ds}}=\frac{-\gamma}{j \omega \mu_{\mathrm{s}}} E_{\mathrm{s}} \mathrm{e}^{\gamma d_{\mathrm{s}}-K_{\mathrm{s}}} \cosh \left(\gamma d_{\mathrm{s}}\right)
\end{array}\right\} .
$$

We neglect the space of the air gap, since owing to the distances $d$ and $d_{\mathrm{s}}$ between the conductors and the air gap, it is possible to take into account the leakages between the rotor and the stator, and this is what is important.

We could equate the fields of the expressions (62) and (63), but the resulting equation would describe a machine without motion which would simply behave as a transformer. Before carrying out this algebraic operation, the fields of the rotor must be modified in order to express its motion.

We shall first write the fields in the moving frame of reference. It is sufficient to add the signs' to all the symbols in equation (63). We shall also change a few indices so as to further particularize the rotor.

After transforming $E_{\mathrm{D}}^{\prime}$ and $H_{\mathrm{D}}^{\prime}$ to the fixed frame of reference by means of the equations (37) and (45), one obtains :

$$
\begin{aligned}
& E_{\mathrm{D}}=\frac{\omega}{\omega^{\prime}} E_{\mathrm{r}}^{\prime} \mathrm{e}^{\gamma^{\prime} d_{\mathrm{r}}^{\prime}}+\frac{j \omega \mu_{\mathrm{r}}^{\prime}}{\gamma^{\prime}} K_{\mathrm{r}}^{\prime} \sinh \left(\gamma^{\prime} d_{\mathrm{r}}^{\prime}\right) \\
& H_{\mathrm{D}}=H_{\mathrm{D}}^{\prime} .
\end{aligned}
$$

These are the expressions that must be set equal to those of equations (63). They may however be simplified. The signs' are unnecessary in a nonrelativistic transformation of the quantities $\mu, \gamma, d$. They are essential for $\omega^{\prime}$ and we shall leave them on $E_{\mathrm{r}}^{\prime}$ and $K_{\mathrm{r}}^{\prime}$ in order to recall, not only that these quantities are inserted in the moving reference frame, but also that they must be transformed in accordance with the mode of excitation of the rotor (sector commutators, ring contacts, etc.) to completely describe the behaviour of the machine. We shall therefore write the equations (64) (65) in the form :

$$
\left.\begin{array}{l}
E_{\mathrm{D}}=\frac{\omega}{\omega^{\prime}} E_{\mathrm{r}}^{\prime} \mathrm{e}^{\gamma d_{\mathrm{r}}}+\frac{j \omega^{\prime} \mu_{\mathrm{r}}}{\gamma} K_{\mathrm{r}}^{\prime} \sinh \left(\gamma d_{\mathrm{r}}\right) \\
H_{\mathrm{D}}=\frac{\gamma}{j \omega^{\prime} \mu_{\mathrm{r}}} E_{\mathrm{r}}^{\prime} \mathrm{e}^{\gamma d_{\mathrm{r}}}+K_{\mathrm{r}}^{\prime} \cosh \left(\gamma d_{\mathrm{r}}\right)
\end{array}\right\}
$$


With equations (63) and (66), there obtains, in assuming that the constants $\mu$ are equal in the stator and rotor :

$$
\begin{gathered}
-E_{\mathrm{s}}=\frac{j \omega \mu}{2 \gamma} K_{\mathrm{s}}+\frac{j \omega \mu}{2 \gamma} \mathrm{e}^{-\gamma \mathrm{D}} K_{\mathrm{r}}^{\prime} \\
-E_{\mathrm{r}}^{\prime}=\frac{j \omega^{\prime} \mu}{2 \gamma} K_{\mathrm{s}} \mathrm{e}^{-\gamma \mathrm{D}}+\frac{j \omega^{\prime} \mu}{2 \gamma} K_{\mathrm{r}}^{\prime} \\
D=d_{\mathrm{r}}+d_{\mathrm{s}} .
\end{gathered}
$$

Assuming now that each winding comprises $N_{\mathrm{s}}$ and $N_{\mathrm{r}}$ conductors, respectively, and that the effective length of the circuits in the direction $z$ is $h$, we obtain from equations (32) and (33) :

$$
\left.\begin{array}{l}
V_{\mathrm{s}}=j \omega \frac{\mu N_{\mathrm{s}}^{2} h}{2 \gamma_{\mathrm{p}}} I_{\mathrm{s}}+j \omega \mu \frac{N_{\mathrm{r}} N_{\mathrm{s}} h}{2 \gamma_{\mathrm{p}}} \mathrm{e}^{-\gamma \mathrm{D}} I_{\mathrm{r}}^{\prime} \\
V_{\mathrm{r}}^{\prime}=j \omega^{\prime} \frac{\mu N_{\mathrm{r}} N_{\mathrm{s}} h}{2 \gamma_{\mathrm{p}}} \mathrm{e}^{-\gamma \mathrm{D}} I_{\mathrm{s}}+j \omega^{\prime} \frac{\mu N_{\mathrm{r}}^{2}}{2 \gamma_{\mathrm{p}}} I_{\mathrm{r}}^{\prime}
\end{array}\right\}
$$

with, obviously :

$$
\omega^{\prime}=\omega-\beta \mu .
$$

The value of $\gamma$ is given by equation (24).

These equations are of the well known form :

$$
\left.\begin{array}{c}
V_{\mathrm{s}}=j \omega L_{\mathrm{s}} I_{\mathrm{s}}+j \omega M I_{\mathrm{r}}^{\prime} \\
V_{\mathrm{r}}^{\prime}=j \omega^{\prime} M I_{\mathrm{s}}+j \omega^{\prime} L_{\mathrm{r}} I_{\mathrm{r}}^{\prime}
\end{array}\right\} .
$$

The prime indices are always kept on the quantities $V_{\mathrm{r}}^{\prime}$ and $I_{\mathrm{r}}^{\prime}$ for the reasons explained above.

Equations (70) can be obtained more rapidly and in a more conventional manner, in starting with considerations of magnetic flux. The calculation given here gives the general method applicable to more complex cases, which permits taking into account the air gap, the dimensions of the frame, etc. We have furthermore obtained the field values in the air gap, which permit an evaluation of the forces and the power fluxes according to paragraph 3 .

6. Equation of the traveling-wave tube. -6.1 . GENERAL FORM OF THE EQUATION. - To give the principle of the method of analysis, we shall diagrammatically represent below the disposition of the elements of a bi-dimensional traveling-wave tube and the topology of the electric field (see figure 5).

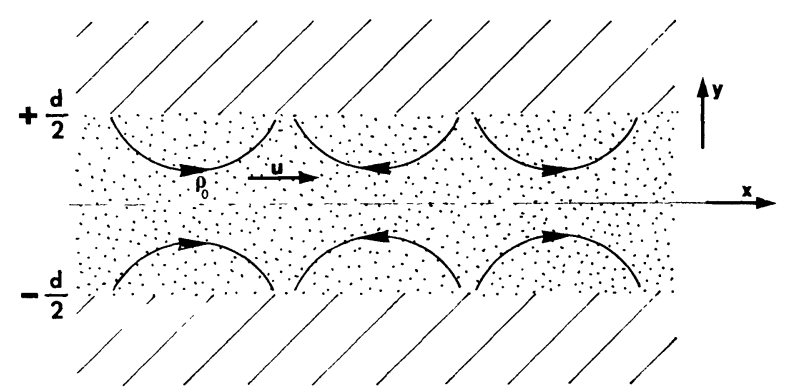

FIG. 5. - Diagram showing the interaction space, the field lines, the symbols.
The bi-dimensional structure has merely the effect of replacing the Bessel functions by hyperbolic functions, which are easier to handle.

The field $E_{y}$ is null on the axis, as is $H_{x}$, according to equation (4). In view of the equations (6') and (30), we have in the inertial system moving with beam :

$$
\left.\begin{array}{rl}
Y^{\prime}(0) & =0 \\
Y^{\prime}\left(\frac{d^{\prime}}{2}\right) & =-\frac{j \omega^{\prime} \varepsilon}{\gamma^{\prime}} \tanh \left(\gamma^{\prime} \frac{d^{\prime}}{2}\right)
\end{array}\right\} .
$$

We employ a very elementary description of the beam, which is contained in the constant $\varepsilon$, whose value, according to equation (5), is :

$$
\varepsilon^{\prime}=\varepsilon_{0}\left(1-\frac{\omega_{\mathrm{p}}^{\prime 2}}{\omega^{\prime 2}}\right)
$$

with

$$
\omega_{\mathrm{p}}^{\prime 2}=\frac{\rho_{0}^{\prime} \mathrm{e}}{m^{\prime} \varepsilon_{0}} .
$$

We obtain the transverse admittance on the upper edge of the beam by means of equation (46) :

$$
Y\left(\frac{d}{2}\right)=-j \frac{\omega \varepsilon_{0}}{\gamma}\left(1-\frac{\omega_{\mathrm{p}}^{2}}{\omega^{\prime 2}}\right) \tanh \left(\gamma \frac{d}{2}\right)
$$

where we have neglected small relativistic corrections.

This admittance must be equated to that presented by the half of the retarding circuit that lies in the region $y>d / 2$.

If $Y_{\mathrm{c}}$ is its admittance, we obtain :

$Y_{\mathrm{c}}\left(\omega, \beta_{0}\right)+\frac{j \omega \varepsilon_{0}}{\gamma} \tanh \left(\gamma \frac{d}{2}\right)=\frac{j \omega \varepsilon_{0}}{\gamma} \frac{\omega_{\mathrm{p}}^{2}}{\omega^{\prime 2}} \tanh \left(\gamma \frac{d}{2}\right)$.

Where all the characteristics of the electron beam are in the right member, and in particular the quantity $\omega_{p}^{2}$, which is proportional to the electronic charge density.

We have here again a transcendental equation, which forms with equation (24) a system having two unknowns, $\beta$ and $\gamma, \omega$ being assumed fixed. To solve it, recourse its usually had [1] to developments limited to the first order of $\omega_{p}^{2}$ (small C approximation, see equations (81) and (82)). Indeed in the absence of the beam, we have :

$$
Y\left(\omega, \beta_{0}\right)+\frac{j \omega \varepsilon_{0}}{\gamma_{0}} \tanh \left(\gamma_{0} \frac{d}{2}\right)=0
$$

and we assume that the presence of the beam results in only a relatively small modification of the value of $\beta$.

We introduce an admittance of the circuit without a beam :

$$
Y(\omega, \beta) \equiv Y_{\mathrm{c}}(\omega, \beta)+\frac{j \omega \varepsilon_{0}}{\gamma} \tanh \left(\gamma \frac{d}{2}\right) .
$$


The calculation of $Y$ is a problem of conventional electromagnetism with no moving circuit. The equation becomes :

$$
Y(\omega, \beta)=\frac{j \omega \varepsilon_{0}}{\gamma} \frac{\omega_{\mathrm{p}}^{2}}{\omega^{\prime 2}} \tanh \left(\gamma \frac{d}{2}\right)
$$

6.2 CaSe « Without SPACE CHARGE ». -- We simply write to the first order of $\beta-\beta_{0}$ :

$\left.\left(\beta-\beta_{0}\right) \frac{\partial Y}{\partial \beta}\right|_{\beta 0}=\frac{j \omega \varepsilon_{0}}{\gamma_{0}} \frac{\omega_{\mathrm{p}}^{2}}{\left(\omega^{\prime}-\beta u\right)^{2}} \tanh \left(\gamma_{0} \frac{d}{2}\right)$.

This is an algebraic equation of the third order of $\beta$. Following Pierce (whose notation for $\beta$ is different), we set :

$$
\beta_{e}=\frac{\omega}{u}
$$

and obtain :

$$
\frac{\beta-\beta_{0}}{\beta_{e}}\left(\frac{\beta-\beta_{e}}{\beta_{e}}\right)^{2}=-C^{3}
$$

defining the parameter $C$ :

$$
C^{3}=\frac{e \rho_{0} u}{m} \frac{\tanh \left(\gamma_{0} d\right)}{j Y_{\beta}^{\prime} \omega^{2}} .
$$

We have made $\omega_{p}^{2}$ again explicit according to equation (5) (Appendix I).

The first factor on the right characterizes the beam, the second the circuit.

The continuous current carried by the beam of width $h$ in the direction $z$, is :

$$
I=\rho_{0} u \mathrm{~d} h .
$$

The notation of Pierce is :

$$
C^{3}=\frac{I K}{4 V}
$$

where $V$ designates the electron-accelerating voltage :

$$
e V=\frac{m u^{2}}{2} \text {. }
$$

The expressions (82) and (84) are identified in setting :

$$
\frac{1}{K}=\frac{1}{2}\left(j \frac{\omega}{u} Y_{\beta}^{\prime}\right) \frac{\omega h}{u} \frac{\omega d}{u} \operatorname{coth}\left(\gamma_{0} d\right) .
$$

The study of the circuit justifies this equality. If the circuit is without losses, $Y$ is purely imaginary.

6.3 CASE WITH SPACE CHARge. - A better approximation is obtained in developing not $Y$ around its root $\beta_{0}$, but $Z=1 / Y$ around its pole, and in keeping only the following term of MacLaurin's development, which term is independent of $\beta-\beta_{0}$ :

$$
Z=\frac{A_{-1}\left(\beta_{0}, \omega\right)}{\beta-\beta_{0}}+A_{0}\left(\beta_{0}, \omega\right)
$$

We have obviously :

$$
A_{-1}=\left[\left.\frac{\partial Y}{\partial \beta}\right|_{\beta_{0}}\right]^{-1}
$$

The equation (78) becomes :

$$
\frac{A_{-1}}{\beta-\beta_{0}}+A_{0}=\frac{\gamma u^{2}}{j \omega \varepsilon_{0}} \frac{\left(\beta-\frac{\omega}{u}\right)^{2}}{\omega_{\mathrm{p}}^{2}} \operatorname{coth}\left(\gamma \frac{d}{2}\right) \text {. }
$$

It can be written :

$\frac{j A_{-1}}{\beta-\beta_{0}}=\frac{\gamma u^{2}}{\omega \varepsilon_{0}} \frac{\operatorname{coth}\left(\gamma \frac{d}{2}\right)}{\omega_{\mathrm{p}}^{2}} \times$
$\times\left[\left(\beta-\frac{\omega}{u}\right)^{2}-\omega_{\mathrm{p}}^{2} j A_{0} \frac{\omega \varepsilon_{0} \tanh \left(\gamma \frac{d}{2}\right)}{\gamma u^{2}}\right]$.

This equation can be identified with the corresponding equation of Pierce in putting :

$$
4 Q \beta_{e}^{2} C^{3}=\omega_{\mathrm{p}}^{2} j A_{0} \frac{\omega \varepsilon_{0} \tanh \left(\gamma \frac{d}{2}\right)}{\gamma u^{2}} .
$$

The electromagnetic theory of the circuit permits a calculation of $A_{0}$ and the obtainment of the exact value of $Q$ for each circuit. The equation with space charge is written :

$$
\frac{\beta-\beta_{0}}{\beta_{e}}\left[\left(\frac{\beta-\beta_{e}}{\beta_{e}}\right)^{2}-4 Q C^{3}\right]=-C^{3} .
$$

When the value of $\beta$ has been obtained, namely :

$$
\beta=\beta_{\mathrm{r}}+j \beta_{\mathrm{i}} .
$$

The space-time variation of any quantity $\psi$ (see equation (1)) is described in accordance with the equation :

$$
\psi=\psi_{0} \mathrm{e}^{j\left(\omega t-\beta_{\mathrm{r}} x\right)} \mathrm{e}^{\beta_{\mathbf{i}} x}
$$

The amplification of power per unit length is then, in decibels :

$$
g=\frac{10 \log _{10} \mathrm{e}^{2 \beta_{\mathrm{i}} l}}{l}=20 \beta_{\mathrm{i}} \operatorname{iog} e=8.7 \beta_{\mathrm{i}} .
$$

In cylindrical geometry, the hyperbolic functions are replaced by modified Bessel functions.

We have thus shown how a single method of analysis and two dual sets of equations can lead to the basic equations of certain rotating machines and microwave tubes. 
PART TWO : list of symbols.

\begin{tabular}{|c|c|}
\hline$U_{n}, V_{n}$ & : Voltages. \\
\hline$\psi$ & : Phase shift between adjacent circuits. \\
\hline $\mathrm{s}, \mathrm{r}$ & : Subscripts for stator, rotor. \\
\hline$L, M$ & : Self-, mutual inductances. \\
\hline $\mathcal{R}, \mathcal{S}$ & $\begin{array}{l}\text { Expressions characterizing the electrical states } \\
\text { in the rotor, the stator, are the coupling } \\
\text { between them. }\end{array}$ \\
\hline$\psi_{0}, \beta_{0}$ & : Particular values of $\psi, \beta$. \\
\hline$\underline{v_{\mathrm{g}}}$ & : Group velocity of a wave. \\
\hline $\bar{W}$ & $\begin{array}{l}\text { : Time-averaged energy stored in a cell of a } \\
\text { periodic circuit. }\end{array}$ \\
\hline $\bar{P}$ & : Time-averaged power carried by the same. \\
\hline$Q$ & $\begin{array}{l}\text { : Pierce's space-charge factor, or its equivalent } \\
\text { in the equation of a rotating machine. }\end{array}$ \\
\hline$F$ & $\begin{array}{l}\text { Dimensionless quantity analogous to Pierce's } \\
C \text { factor, characterizing some types of rotat- } \\
\text { ing machines. }\end{array}$ \\
\hline$x, y$ & $\begin{array}{l}\text { (If not space coordinates) dimensionless velo- } \\
\text { city slip and gain parameters in an amplifying } \\
\text { rotating machine. }\end{array}$ \\
\hline$\tau$ & : Normalized characteristic time of a rotor. \\
\hline & : Power gain of an amplifier (in decibels). \\
\hline$R_{\mathrm{s}}$ & $\begin{array}{l}\text { : Resistances of elements in the stator or the } \\
\text { rotor; also, reflection coefficients at the } \\
\text { output and input of a stator. }\end{array}$ \\
\hline
\end{tabular}

1. Differences between electrical machines and microwave tubes. - Having found a certain number of resemblances between rotating machines and several types of microwave electronic devices, we have given, in Part One of this paper, a formalism that translates in a unique manner, but with two variants (magnetic and electric), the transformation of the fields, the currents and the voltages in these apparatus. We thus established formulas and a method of calculation which permitted to obtain two results as different as, on one hand, two circuit equations which may serve as a basis for the theory of most rotating machines, and on the other hand, the equation which permits the calculation of the amplification in traveling-wave tubes.

It may seem obvious at this point that most microwave devices should have their counterpart in rotating or linear machinery, and vice versa. This is, however, far from being the general case : magnetrons and alternators are very similar, but this is essentially where the analogy stops among existing apparatus. Wave transmission amplifiers of the electromechanical type (and other types) were patented a long time ago [2]. They have, to our knowledge, not yet found practical application, however. It is true that the devices, as patented, present serious drawbacks as compared to existing machinery. Recently, the author has proposed several types of machines that seem more promising to him [9-12].

Before describing these machines, let us examine in more details the differences between electrical machines and microwave tubes.

Some already became apparent during the developments in the first part of this paper :

1.1 The coupling between the two parts of the device is electric in one case, magnetic in the other.

1.2 The fields are exactly periodic in machines, most of which are closed on a circle, whereas their amplitude varies, in general, along, the length of microwave tubes (except in the case of the magnetron, which as already stated, is the microwave tube closest to rotating machines). Consequently, in rotating machines, the poles of the same phase can be interconnected.

1.3 In magnetic machines, current is taken from each of the pole ?windings. These currents are then connected in different manners and grouped in a small number of conductors characterized by their phase and connected to a network. The electrical energy stored is considerably less than the magnetic energy. High fields are obtained in multiplying the turns of the windings.

There is no analogous procedure for increasing the induction flux in the electronic devices. The fields are increased in microwave tubes by converting the electrical energy produced into magnetic energy, by means of the inductive parts of the microwave circuit. The increase in the inductance serves both to increase the intensity of the electric field and to retard the wave, the propagation being ensured by the conversion of electric energy into magnetic energy and vice versa. The two energies are stored in equal quantities, on the average. Microwave tubes generally have a single output placed at one end of the circuit or propagation line and, if they are amplifiers, a single input at the other end.

1.4 Rotors are rigid, electron beams are not. Each element of volume of the moving parts is subjected to electromagnetic forces and to mechanical forces in machines; in beams, it is the force of inertia that balances the electromagnetic forces.

At each instant, a conductor in the inductor of an alternator receives from the rotor of which it is a part the mechanical energy that it converts into electrical energy, without changing velocity and without modification of the phase of the rotating field in which it is located. In traveling-wave tubes of 0 type (ordinary), the electrons are retarded.

Since their kinetic energy is the sole source of electromagnetic energy, the phase necessarily varies. In order to put the electrons into the correct phase, they must be injected at a velocity higher than that of the wave, so that they remain approximately in phase while being retarded, but it will be understood that the quantity of kinetic energy that they may lose before falling, into an accelerating phase is limited. In the magnetron and its derivatives, traveling-wave tubes of the $M$ type, the question is different : the electrons move in transverse electric and magnetic fields and the first field gives up 
energy to them while they accompany the wave at a roughly constant velocity. Magnetrons are again, in most respects, the closest to alternators ; apart from the dual difference, only the method of energy pick-off is different, but not essential.

1.5 A consequence of the foregoing statement : there are not mechanical energy losses in electric machines, apart from friction, with the restriction of prolonged operation (upon each stoppage the kinetic energy of the rotor is lost). In electron tubes, a more or less large part of the kinetic energy that remains in the electrons when they leave the delay circuit is necessarily lost.

1.6 In rotating machines, there may be a highefficiency linear operating condition. Microwave tubes are always non-linear in their high-efficiency regions.

2. Principles and basic equations of new amplifying machines. - It follows from these considerations that there is nothing to prevent the construction of a rotating machine operating, for example, as a wideband amplifier over the frequency domain in which an alternator is practically constructible. To achieve this, the circuits of the stator must be associated with capacitances so as to form a periodic filter, consituting a transmission line, provided with an input and an output which may be those of the amplifier system. Figure $6 a$ relates to a low-pass filter circuit if the transverse admittance is a capacitance. The filter has a roughly constant velocity in the low-frequency part of its passband, which permits operation over a wide band of frequencies, since the velocity of the rotor and the velocity of the phase must remain close to each other.

The circuit equations are written in using the notations of the figure 6 , where $\Psi$ is the phase shift per cell and in leaving out the direct relations between $V_{n}$ and $I_{n}$ which bring in the rotor:

$$
\begin{aligned}
V_{n}= & U_{n-1}-U_{n}=U_{n}\left(\mathrm{e}^{j \Psi}-1\right) \\
& I_{n}\left(1-\mathrm{e}^{-j \Psi}\right)=Y U_{n}
\end{aligned}
$$

with

$$
\Psi=\beta p .
$$

We shall here limit ourselves to the case where the transverse admittance is purely capacitive. We then obtain :

$$
V_{n}=-\frac{I_{n}}{j \omega C} 4 \sin ^{2} \frac{\beta p}{2}
$$

Two important quantities are the following characteristic impedances :

$$
\begin{aligned}
& Z_{T} \equiv \frac{U_{n}+U_{n-1}}{2 I_{n}}-j \frac{V_{n}}{2 I_{n}} \cot \frac{\Psi}{2}=\frac{\sin \beta p}{\omega C} \\
& Z_{n} \equiv \frac{2 U_{n}}{I_{n}+I_{n+1}}=\frac{V_{n}}{j I_{n} \sin \Psi}=\frac{2}{\sim} \tan \frac{\beta p}{2} .
\end{aligned}
$$
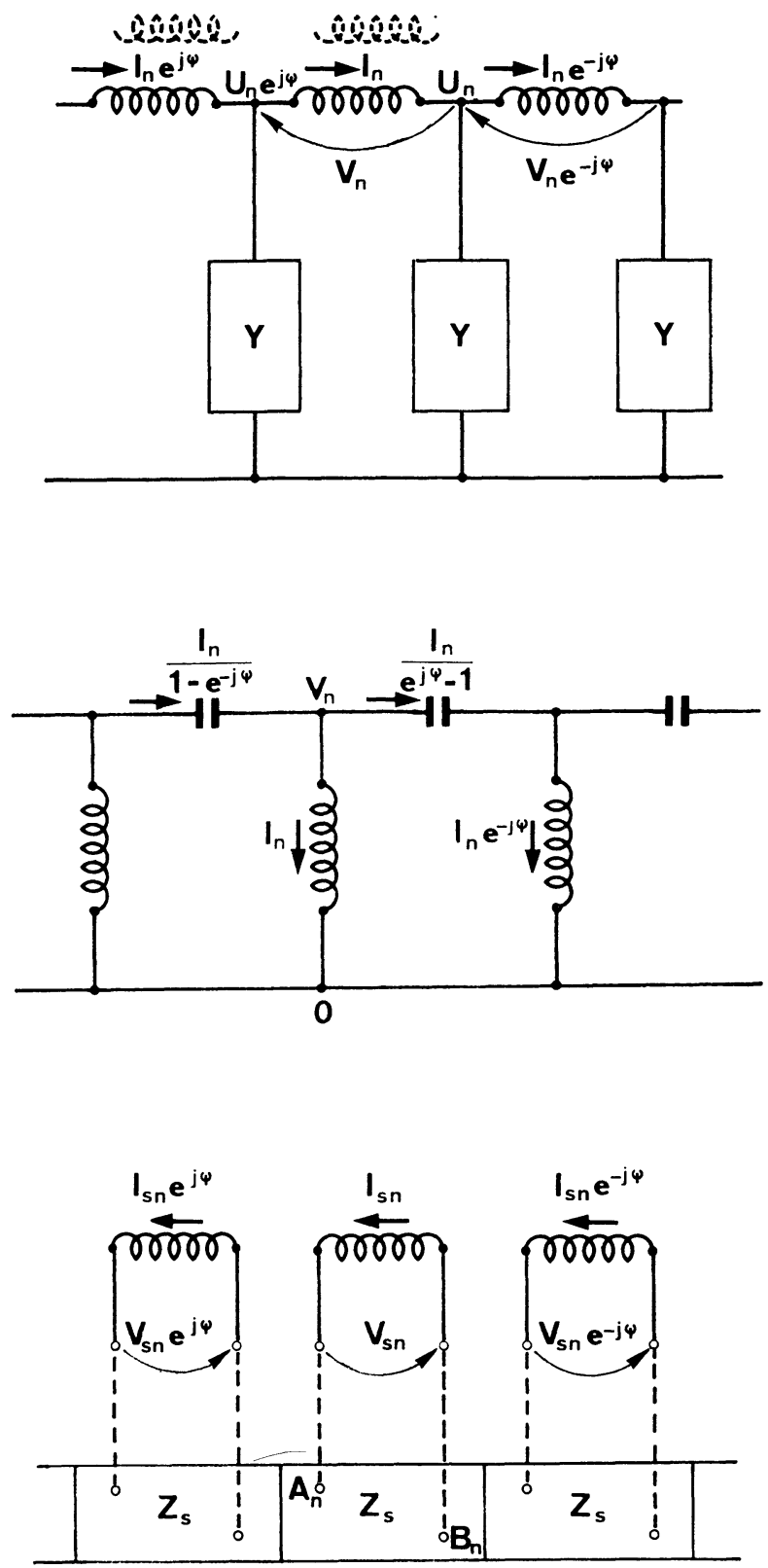

Fig. 6. - A diagram of stators connected as a delay line ; symbols.

Many circuits are possible. In the case shown in figure $6 b$ which corresponds to a high-pass filter, we have :

$$
V_{n}=\frac{j}{4 C \omega \sin ^{2} \frac{\beta p}{2}} I_{n} .
$$

Irrespective of the connection, the equations define the filter chain furnishing an expression of the form

$$
V_{\mathrm{s}}=-Z_{\mathrm{s}}(\omega, \beta) I_{\mathrm{s}} \text {. }
$$

This expression is established without taking into account the structure of the stator windings, or its coupling to the rotor, but only the circuit to which these windings are connected.

A second relation between $V_{\mathrm{s}}$ and $I_{\mathrm{s}}$ will be obtained, 
which is a characteristic of the windings and their coupling to the rotor, based on the equations (70) of Part One.

Let $Z_{\mathrm{r}}$ be the impedance related to the windings of the rotor. This will be, for example, a capacitance, a resistance or a filter network, as in the stator. In the latter case, $Z_{\mathrm{r}}$ is a function of $\Psi$. In any case. if $Z_{\mathrm{r}}$ is not a pure resistance, it is a function of the frequency, the value of which is $\omega^{\prime}$ since the rotor is in motion. We shall therefore write $Z_{\mathbf{r}}\left(\omega^{\prime}\right)$ or $Z_{\mathbf{r}}^{\prime}$. We then obtain :

$$
V_{\mathrm{s}}=\left[j L_{\mathrm{s}} \omega+\frac{M^{2} \omega \omega^{\prime}}{j L_{\mathrm{r}} \omega^{\prime}+Z_{\mathrm{r}}^{\prime}}\right] I_{\mathrm{s}} .
$$

This equation no longer contains the voltages and current of the rotor, whose role appears only in the second term in the bracket. It permits, with equation (8), describing the behaviour of the machine seen as a filter chain, which we could later connect to a source, a utilization network, etc.

To study the circuit, the impedance $Z_{\mathrm{s}}$ being specified as a function of $\omega$ and $\beta$, we shall in general start to look for the values of $\Psi$ that are compatible, for each $\omega$, with equations (8) and (9). (Sometimes, on the other hand, we shall look for the values of $\omega$ for a given value of $\Psi$ ). These values will in general be sufficient to obtain the magnitude of the amplification, the conditions of stability, etc.

The problem is first to study the dispersion equation (relation between $\omega$ and $\beta$ ) for a complex propagation system. Then, as each value of $\Psi$, for a given value of $\omega$, corresponds to a system of voltages and currents that are propagated along the filter, the current in each arm to the circuit is considered to be the sum of as many components as there are solutions for $\Psi$, each component obeying a phase shift law conforming to equation (1) (paragraph 3, Part One) with the corresponding value of $\Psi$. One can then write the equations at the input and output points ot the stator. It is also necessary to write the conditions concerning the rotor at the corresponding points, which may be more delicate.

The equation in $\Psi$, taken from (8) and (9), is the following :

$$
\left[Z_{\mathrm{s}}+j L_{\mathrm{s}} \omega\right]\left[Z_{\mathrm{r}}^{\prime}+j L_{\mathrm{r}} \omega^{\prime}\right]+M^{2} \omega \omega^{\prime}=0 .
$$

It can be put into the form of the general equation :

$\left[\frac{\omega^{\prime}}{\omega}-j \frac{Z_{\mathrm{r}}^{\prime}}{L_{\mathrm{r}} \omega}\right]\left[Z_{\mathrm{s}}+j L_{\mathrm{s}} \omega\left(1-\frac{M^{2}}{L_{\mathrm{r}} L_{\mathrm{s}}}\right)\right]=-Z_{\mathrm{r}} \frac{M^{2}}{L_{\mathrm{r}}^{2}}$.

We can again write in the condensed form :

$$
\mathfrak{R S}=\mathcal{C}
$$

where $\mathcal{R}$ depends on the characteristics of the rotor, $\delta$ on the characteristics of the stator and $\mathcal{C}$ is a coupling term. This equation is transcendental, owing to the expression of $Z_{\mathrm{s}}$. If the amplification per cell is not very large, an algebraic equation can be substituted for it. This supposition does not severely limit in practice the amplification of the machine, which may have many cells.

3. Approximate solution of the general equation (weak amplification). - It will be assumed that the rotor modifies but little the local conditions in the stator, which behaves roughly as a filter. An extreme case will first be examined :

3.1 ROTOR SHORT-CIRCUITED. - If its resistance is truly negligible, we have

$$
\begin{aligned}
Z_{\mathrm{r}}^{\prime} & =0 \\
\omega^{\prime} \delta & =0 .
\end{aligned}
$$

The second equation indicates that there are two solutions, corresponding to equations 14 and 15 .

$$
\omega^{\prime}=0 \quad \beta=\frac{\omega}{u} .
$$

This solution is attached to the rotor, which can only contain direct currents.

$$
\mathcal{S} \equiv Z_{\mathrm{s}}+j L_{\mathrm{s}} \omega\left(1-\frac{M^{2}}{L_{\mathrm{r}} I_{\mathrm{s}}}\right)=0 .
$$

$Z_{\mathrm{s}}$ being a function of $\omega$ and $\Psi$, this equation describes the filter chain constituted by the rotor. In simple cases, it contains only one root in $\sin ^{2} \frac{\Psi}{2}$, to which there corresponds a value that we shall write, as in paragraph 6, Part One :

$$
\Psi_{0} \equiv \beta_{0} p
$$

It can be seen that there is no coupling between the rotor and the stator if $Z_{\mathrm{r}}^{\prime}$ is null.

3. 2 WEAK COUPLING. - Assuming that $\beta$ is scarcely different from $\beta_{0}$ we can write :

$$
\mathcal{S}(\omega, \beta) \simeq \mathrm{S}\left(\omega, \beta_{0}\right)+\left(\beta-\beta_{0}\right) \frac{\partial \mathrm{S}}{\partial \beta_{0}} .
$$

The first term of the second member is null according to equation (15), and we can write :

$\left[\frac{\omega^{\prime}}{\omega}-j \frac{Z_{\mathrm{r}}^{\prime}}{I_{\mathrm{r}} \omega}\right] \frac{\left(\beta-\beta_{0}\right)}{\omega / u}=-Z_{\mathrm{r}}^{\prime} \frac{M^{2}}{L_{\mathrm{r}}^{2}}\left[\frac{\omega}{u} \frac{\partial \mathrm{S}}{\partial \beta_{0}}\right]^{-1}$

where $\beta$ is contained on one hand, explicitly and on the other hand, in $\omega^{\prime}$ and therefore in $Z_{\mathrm{r}}^{\prime}$. The degree of the equation and the number of roots solely depend on $Z_{r}^{\prime}$, which may contain $\omega^{\prime}$ (we have assumed that the stator can guide only one mode, characterized by a value of $\Psi_{0}$ ). The second member may be transformed by noting that the dispersion equation (relation between $\beta_{0}$ and $\omega$ ) of the stator, when the rotor in shortcircuited, is equation (15), which results in :

$$
\frac{\partial \mathcal{S}}{\partial \omega} \mathrm{d} \omega+\frac{\partial \mathcal{S}}{\partial \beta_{0}} \mathrm{~d} \beta=0 .
$$


Now, the group (or energy) velocity is

$$
v_{\mathrm{g}}=\frac{\mathrm{d} \omega}{\mathrm{d} \beta} .
$$

Moreover :

$$
\frac{\partial \mathcal{S}}{\partial \omega}=\frac{\mathrm{d} Z_{\mathrm{s}}}{\mathrm{d} \omega}+j L_{\mathrm{s}}\left(1-\frac{M^{2}}{L_{\mathrm{r}} L_{\mathrm{s}}}\right) .
$$

If the impedance $Z_{\mathrm{s}}$ is without losses, or has low losses, the energy stored in a cell of the circuit is, according to a general theorem of pure reactances:

$$
\begin{aligned}
\bar{W}=-\frac{j}{4} \frac{\partial \mathrm{S}}{\partial \omega} I_{\mathrm{s}} I_{\mathrm{s}}^{*}= & -\frac{j}{4} \frac{\mathrm{d} Z_{\mathrm{s}}}{\mathrm{d} \omega} I_{\mathrm{s}} I_{\mathrm{s}}^{*}+ \\
& +L_{\mathrm{s}} I_{\mathrm{s}} I_{\mathrm{s}}^{*}\left(1-\frac{M^{2}}{L_{\mathrm{r}} L_{\mathrm{s}}}\right) .
\end{aligned}
$$

We therefore have :

$$
\frac{\partial \mathrm{S}}{\partial \beta_{0}}=-4 j \frac{\bar{W} v_{\mathrm{g}}}{I_{\mathrm{s}} I_{\mathrm{s}}^{*}}=-4 j \frac{\bar{P} p}{I_{\mathrm{s}} I_{\mathrm{s}}^{*}}
$$

where $\bar{P}$ is the power transported by the stator under the conditions indicated above.

Equation (18) can therefore be written :

$$
\left[\frac{\omega^{\prime}}{\omega}-j \frac{Z_{\mathrm{r}}^{\prime}}{L_{\mathrm{r}} \omega}\right]\left[\frac{\beta-\beta_{0}}{\omega / u}\right]=-\frac{j}{4} \frac{Z_{\mathrm{r}}^{\prime} I_{\mathrm{s}} I_{\mathrm{s}}^{*}}{\bar{P}} \frac{M^{2}}{L_{\mathrm{r}}^{2}} \frac{1}{\omega p / u} \text {. }
$$

Equations (23) and (24) are completely analogous to the equations for traveling-wave tubes. The quantity $\bar{P} / 2 I_{\mathrm{s}} I_{\mathrm{s}}^{*}$ is very close to Pierce's coupling impedance.

However, it should be mentioned that the approximation of equation (17), which corresponds to the approximation $C$ small of the traveling-wave tubes, could be completely unjustified in certain rotating machines, since the coupling of conventional machines is much stronger than in traveling-wave tubes. It is essential to correctly solve the transcendental equation (11).

4. Dual amplifiers of Microwave tubes [2,9]. - The interest of placing capacitors $C_{\mathrm{r}}$ at the terminals of the windings of the rotor has previously been shown. We then have :

$$
Z_{\mathrm{r}}^{\prime}=\frac{1}{j C_{\mathrm{r}} \omega^{\prime}}
$$

and irrespective of the structure of the filter of the delay line of the stator, equation (24) takes the form :

$$
\left(\frac{\omega^{\prime 2}}{\omega^{2}}-\frac{\omega_{\mathrm{r}}^{2}}{\omega^{2}}\right) \frac{\beta-\beta_{0}}{\omega / u}=-\frac{I_{\mathrm{s}} I_{\mathrm{s}}^{*}}{4 \bar{P}} \frac{1}{C_{\mathrm{r}} \omega} \frac{M^{2}}{L_{\mathrm{r}}^{2}} \frac{u}{\omega p} .
$$

It is reduced to the classical form of the equation for a traveling-wave tube by setting :

$$
\beta_{\mathrm{r}}=\frac{\omega}{u}
$$

$$
\begin{gathered}
C=\left(\frac{I_{\mathrm{s}} I_{\mathrm{s}}^{*}}{4 \bar{P}} \frac{1}{C_{\mathrm{r}} \omega} \frac{M^{2}}{L_{\mathrm{r}}^{2}} \frac{u}{\omega p}\right)^{1 / 3} \\
4 Q C^{3}=\frac{\omega_{\mathrm{r}}^{2} u^{2}}{\omega^{2}}
\end{gathered}
$$

hence,

$$
\frac{\beta-\beta_{0}}{\beta_{\mathrm{r}} C}\left[\frac{\beta-\beta_{\mathrm{r}}}{\beta_{\mathrm{r}} C}-4 Q C\right]=-1 .
$$

This equation is sufficient to show that all the microwave systems-direct wave or inverse-wave amplifiers, tunable oscillators, etc., have their counterparts in these new machines, at least theoretically.

5. Systems having a resistive rotor [10]. - A machine much closer to the prior art will be obtained by placing a resistance at the terminals of each winding of the rotor. In fact, all the systems employed in asynchronous machines are suitable for the rotor. We have :

$$
Z_{\mathrm{r}}^{\prime}=R_{\mathrm{r}}
$$

Setting

$$
F=\frac{I_{\mathrm{s}} I_{\mathrm{s}}^{*}}{4 \bar{P}} \frac{M^{2} \omega}{L_{\mathrm{r}}} \frac{u}{\omega p}
$$

we obtain, with equation (24), an equation analogous to (30):

$$
\left[\frac{\beta-\frac{\omega}{u}}{\frac{\omega}{u} F}+j \frac{R_{\mathrm{r}}}{L_{\mathrm{r}} \omega}\right]\left[\frac{\beta-\frac{\omega}{u}}{\frac{\omega}{u} F}\right]=j \frac{R_{\mathrm{r}}}{F L_{\mathrm{r}} \omega} .
$$

If the stator is connected as a low-pass filter (Fig. 6), we have, from equations (1) to (6) :

$$
\frac{I_{\mathrm{s}} I_{\mathrm{s}}^{*}}{2 \bar{P}}=\frac{2 \tan \frac{\Psi}{2}}{L_{\mathrm{s}} \omega\left(1-\frac{M^{2}}{L_{\mathrm{r}} L_{\mathrm{s}}}\right)}
$$

and

$$
F=\frac{\frac{M^{2}}{L_{\mathrm{r}} L_{\mathrm{s}}}}{1-\frac{M^{2}}{L_{\mathrm{r}} L_{\mathrm{s}}}} \frac{u}{\omega p} \tan \frac{\Psi}{2} .
$$

In the low frequency part of the passband we have :

$$
\frac{u}{\omega p} \tan \frac{\Psi}{2} \approx \frac{1}{2} \frac{\beta_{0} u}{\omega} .
$$

Note that equation (34) may be employed in the same way to calculate the parameter $C$ (Eq. (28)) of paragraph 4. 
We now define dimensionless quantities :

$$
\begin{aligned}
& z \equiv x+j y=\frac{\beta-\frac{\omega}{u}}{\frac{\omega}{u} F} \\
& z_{0} \equiv x_{0}+j y_{0}=\frac{\beta_{0}-\frac{\omega}{u}}{\frac{\omega}{u} F} \\
& \tau=\frac{F L_{\mathrm{r}} \omega}{R_{\mathrm{r}}}
\end{aligned}
$$

and obtain :

$$
\left[z+\frac{j}{\tau}\right]\left[z-z_{0}\right]=\frac{j}{\tau} .
$$

The quantity $\beta_{0} u / \omega$ is directly releated to the slip and can never be very different from $1 . F$ is the parameter of the coupling between the rotor and stator and serves as a unit for the reduced quantities, which are :

$x_{0}$, parameter of synchronism between the wave of the non-coupled stator $(F=0)$ and the rotor. It is positive when the rotor is hypersynchronous. It may be termed a parameter of slip without coupling.

$y_{0}$; negative parameter measuring the losses in the stator or the load distributed on a segment of the latter (see below).

$x$, parameter of synchronism between the wave of coupled stator $(F \neq 0)$ and the rotor. It is positive when the rotor is hypersynchronous. It may be termed a parameter of slip with coupling.

$y$, parameter of amplification $(y>0)$ or attenuation $(y<0)$ of the currents and the voltages along the stator.

$\tau$, a parameter inversely proportional to the ohmic losses in the rotor, and proportional to the relaxation time of the currents in the latter.

As equation (40) is of the second degree, it always has two complex roots of which one always corresponds to an attenuated wave. The other wave may be amplified, in particular when $z_{0}$ is close to 0 . At first, it is the positive values of $y$ that interest us.

The nature of the solutions indicates an exponential gain along the stator. The amplified wave varies as:

$$
\exp -j \frac{\omega}{u}(1+z F) p n
$$

where $p$ is the distance between the poles and $n$ the number that designates the poles. For $N$ poles, the ratio of the amplitudes of the amplified wave at the input and at the output is :

$$
\exp \frac{\omega p}{u} y N
$$

It can be seen that, if the operation is roughly synchronous, the argument of the exponential is the product of $y$ and the total phase shift.
The power gain in decibels is :

$$
G=8.7 \frac{\omega p}{u} N y
$$

It can be seen that, if the total phase shift is $10 \pi$, a gain of $27 \mathrm{~dB}$ can be obtained, even if $y$ does not exceed 0.1 . The gain in fact, is decreased by a few decibels by the conditions of excitation at the input, since

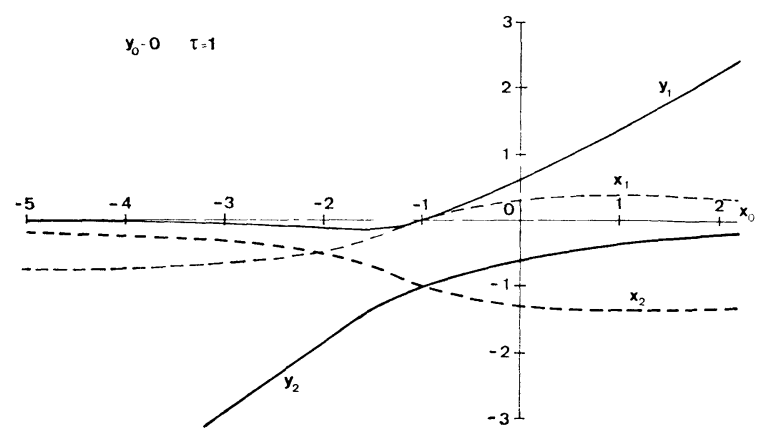

(a)

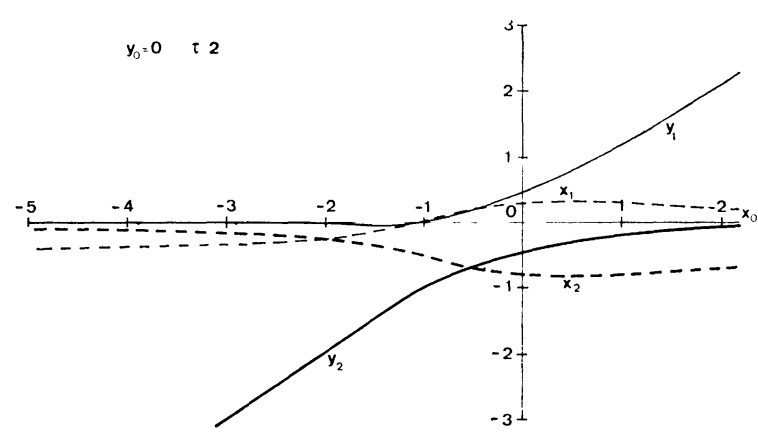

(b)

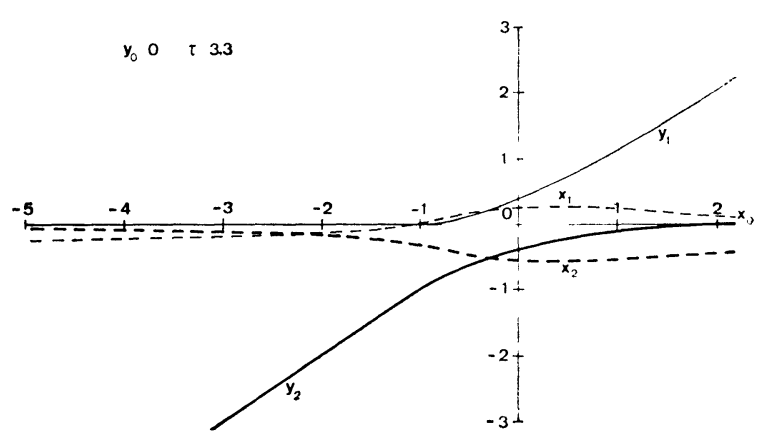

(c)

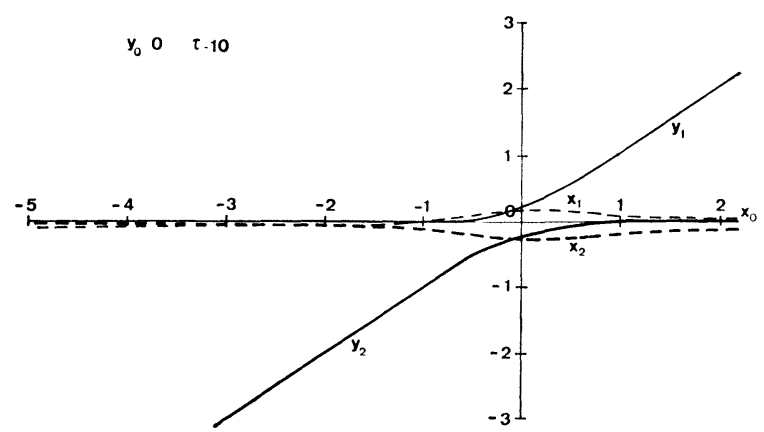

(d)

Fig. $7 a, b, c, d$. - Curves pertaining to the operation of a lossy rotor amplifier. 
the attenuated wave, which disappears rapidly, is excited as well as the amplified wave. The real gain may be $20 \mathrm{~dB}$.

All these problems, treated in great detail in the theory of traveling-wave tubes, may be treated here by the same methods.

Figures $7 a-d$ give the numerical values of the quantities obtained by the solution of (40), assuming the ohmic losses in the stator to be negligible. It is thus shown that, in this case, there is for each value of $\tau$ a particular value of $x_{0}$ that ensures the maximum value of $y_{1}$, that is to say the maximum amplification. This value is :

$$
x_{00}=\frac{1}{\tau}
$$

Figure 8 gives the values of $y_{1}, y_{2}, x_{1}$ and $x_{2}$ for this value of $x_{0}$. Figure 9 gives the same quantities for

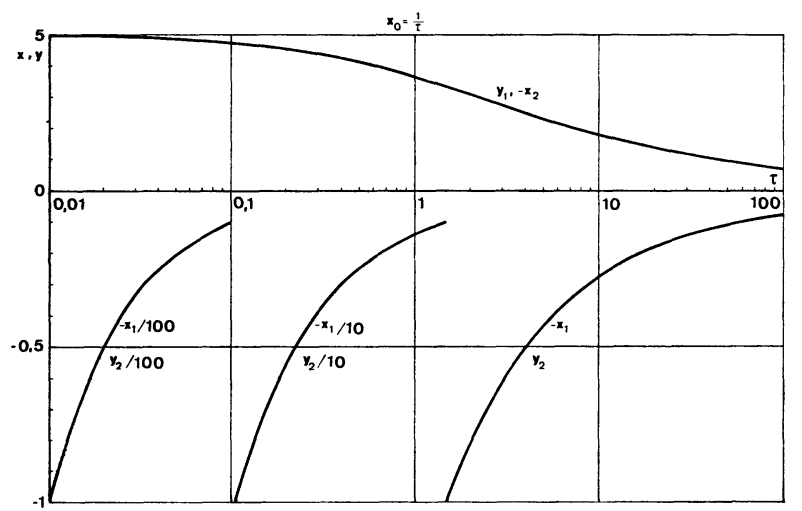

FIG. 8. - Curves pertaining to the optimized operation of a lossy rotor amplifier.

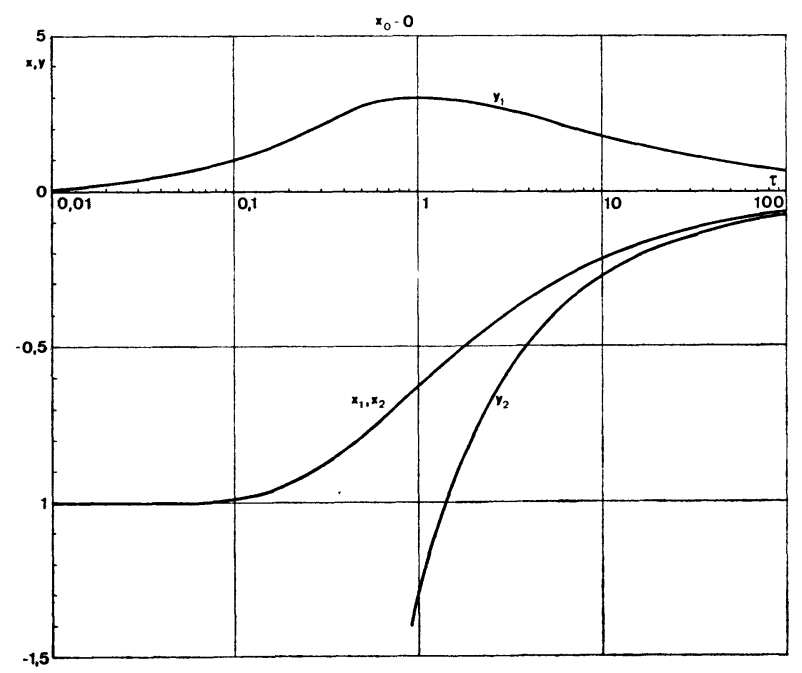

Fig. 9. - Curves showing the influence of the losses in the rotor on the power gain.

$x_{0}=0$. For high values of $\tau$, there is little difference between the two cases. If $\tau$ is small, a large amplification may be obtained, provided that $x_{1}$ is very high (large slip), but the efficiency is then very low.
6. Generalities concerning the characteristics of these new machines. - 6.1 BASIC STATOR AND ROTOR MODIFICATIONS. - The machines analysed in the preceding sections comprise a stator, rather fundamentally different from that of a conventional rotating machine, and a rotor, which may be modified by the addition of capacitors but which, at least in the present state of the art, will be preferably a conventional rotor of an asynchronous machine. It is connected to a source of mechanical energy and interacts with the stator through the air gap by induction, but it is not directly connected to the sources of current and voltage.

On the other hand, the stator is connected if desired, to a source of current furnishing the input signal and in any case, at the output, to a useful load. It has the structure of a periodic filter or power delay line, which gets active by the interaction with the rotor. It has been seen in paragraph 3.5 that there is no need for a considerable modification of the propagation constants, to obtain a considerable amplifying effect : a power gain of 100 in a circuit comprising five cycles with a value of $y$ of 0.1 , that is to say, a variation of the propagation constant of $10 \%$ in absolute value. When the gain equation has been solved, the operation of the machine can be analysed with the following simplified model : only the stator is considered, and it behaves as a periodic filter section in which the rotor produces an active effect for a signal propagated in the direction of the rotor, whereas this effect is negligible in the other direction of propagation.

6.2 OTHER MODIFICATIONS OF THE STATOR. - To really obtain a traveling-wave amplifier, several conditions must still be satisfied :

6.2.1 Interrupt the circle of the stator at a point of its circumference so as to produce an (see Fig. 10)
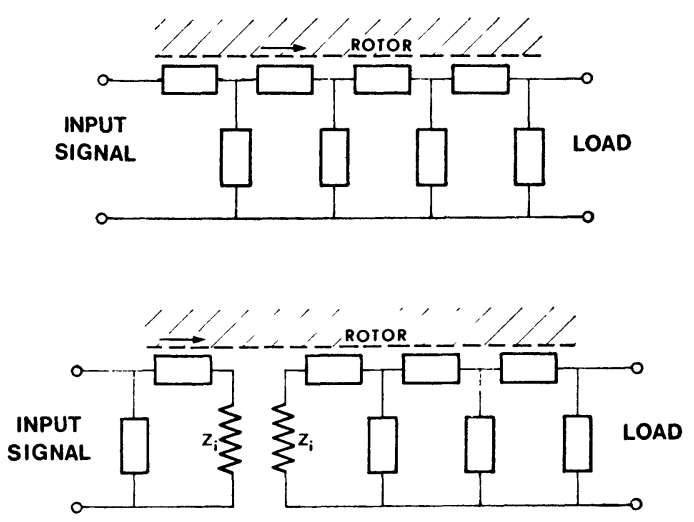

FIG. 10. - Diagrams illustrating the principle of an amplifier and of a stabilized amplifier.

input and an output. This presents no particular difficulty, but creates a mechanical asymmetry that will perhaps have to be compensated for.

6.2.2 Introduce attenuating or decoupling means, so as to avoid reflections of the wave propagated 
between the input and output. This does not seem to present any difficulty, since the techniques employed in microwaves tubes are even easier to employ here.

6.2.3 Damp the oscillations present in the circuits of the rotor when they travel beyond the output of the amplifier. If they still contained oscillations when they were again presented at the input of the stator circuit, a reaction of the output on the input of the amplifier would result, which would inevitably produce undesired oscillations. Means for damping these oscillations have been indicated in the patents.

The amplifying machines just described can be compared to alternators in which the excitation function of the inductor would be performed by the capacitances of the rotor, progressively charged by the windings of the rotor itself. As the oscillation frequency of the circuits of the rotor is rather low, a dc current supply would thus be simulated.

A filter chain like the stator is characterized at each frequency by an impedance termed an iterative or characteristic impedance. If the circuit load does not have this impedance, part $\left(R_{\mathrm{s}}\right)$ of the energy will be reflected toward the input, where it may undergo a new reflection $\left(R_{\mathrm{r}}\right)$. If $g$ is the amplitude gain, there will be an oscillation condition :

$$
\left|g R_{\mathrm{r}} R_{\mathrm{s}}\right|>1
$$

provided that the phase permits this. The filter section, however, has several cycles, and amplification exists over a rather wide frequency interval, in which the impedance of the load and that of the filter vary in a different manner. It is always necessary to stabilize the circuit, which is done, like in traveling-wave tubes, by dividing the filter into two sections matched to their iterative impedance (Fig. 10). This sectioning is made at a sufficient distance from the output to ensure that the power in the filter is still small, otherwise, the energy efficiency would be reduced.

6.3 PowER, ENERGY DENSITY, DIMENSIONS. - In a periodic filter, the mean energy $(\bar{W})$ stored by each cell is related to the mean power $(\bar{P})$ carried along by a traveling wave :

$$
\bar{P}=\bar{W} \frac{\mathrm{d} \omega}{\mathrm{d} \Psi}=\frac{\bar{W}}{p} v_{\mathrm{g}}
$$

where $\Psi$ and $p$ are, as before, the unit phase shift and the geometric length of each cell, and $v_{\mathrm{g}}$ the group velocity. The latter is lower than the phase velocity (except in special cases), which is close to the peripheral velocity of the rotor. For example, if the stator carries a power of $100 \mathrm{~kW}$ at a velocity of $200 \mathrm{~m} / \mathrm{s}$, the energy stored is at least 500 joules per linear meter of the stator. The energies stored in commercially available capacitors having plastic insulation can reach 10 joules/liter. Consequently, 50 liters of capacitors would be required per meter, that is, a section of about $500 \mathrm{sq} \mathrm{cm}$. The large dimensions thus reached for the capacitors (and for the magnetic circuit) are due to the fact that the whole of the output power is furnished to the load through a single cell, namely that which comprises the last pole.

Theoretically, the elements of the stator could be given volumes which vary in accordance with their place in the filter chain, while conserving their linear electrical characteristics. This solution may be partly applied, preferably with an output distributed among a plurality of cells, which will be discussed below.

6.4 EFFICIENCY. - The fact that the power passes through all the output cells reduces the efficiency, but the higher the power gain per cell, the less this is so.

Each cell may be given a quality factor, $Q$, that enables the power of the losses $P_{p}$ in this cell to be calculated :

$$
P_{\mathrm{p}}=\frac{\omega \bar{W}}{Q}=\frac{\bar{P}}{Q} \frac{\omega p}{v_{\mathrm{g}}} .
$$

If $g$ is the power gain per cell, the power generated in this cell is roughly :

$$
P_{0}=\bar{P}\left(\sqrt{g}-\frac{1}{\sqrt{g}}\right)
$$

hence

$$
\frac{P_{\mathrm{p}}}{P_{0}}=\frac{1}{Q} \frac{\omega p}{v_{\mathrm{g}}} \frac{1}{\left(\sqrt{g}-\frac{1}{\sqrt{g}}\right)}
$$

In general, an effort will be made to have a high group velocity, that is to say, a wideband filter. As the efficiency is lower than in conventional machines, it will be necessary in this respect to employ an iron of a high $\mu$ in order to reduce the number of ampere-turns in the stator poles.

The compromise between volume and efficiency will therefore be even more critical than in conventional machines.

6.5 Machines haVing A Distributed LOAD. - The drawbacks of these new machines with respect to their volume and efficiency may be considerably reduced by loading not only the last cell but a theoretically unlimited number of cells located before it. It will be shown that there exists in these cells, a condition of operation without variation of amplitude from cell to cell. This part of the machine therefore, can have characteristics very close to those of conventional machines. It must be preceded by a section of variable amplitude, that produces a first amplification of the signal and which has no need to be of large size.

Each of the loaded cells would furnish the same voltages and currents but with a phase shift. Certain utilizations tolerate this phase shift, either because they have several roughly balanced and independent stations, or because the current is rectified. Otherwise, the various sources must be put back into phase, which requires filters but again allows a saving in volume at the cost of a limitation of the operating frequency band. 
The analysis of this operation is made with the equations of paragraph 3.5. The load on each cell is represented by an attenuation of the stator non-excited by the rotor, which is expressed by giving a negative value to $y_{0}$, and the solutions are sought without amplification $(y=0)$. We find :

$$
\tau x=-\frac{1}{2 y_{0}} \pm \sqrt{\frac{1}{4 \cdot !_{0}^{2}}-1} .
$$

As $y_{0}$ is negative, the root with the - sign corresponds to a lower value of the slip (given by $x$ ) and therefore to a higher efficiency.

This condition of operation is only possible if the cells are not overloaded, i. e., $\left|y_{0}\right|<\frac{1}{2}$.

This machine would appear to be of particular interest, since the part without gain would behave almost exactly as a conventional machine. The circumference would comprise a small modulating sector, serving to excite the rotor at the chosen frequency and amplitude, and a more extensive sector comprising as many outputs as there are poles and having a conventional structure (see Fig. 11).

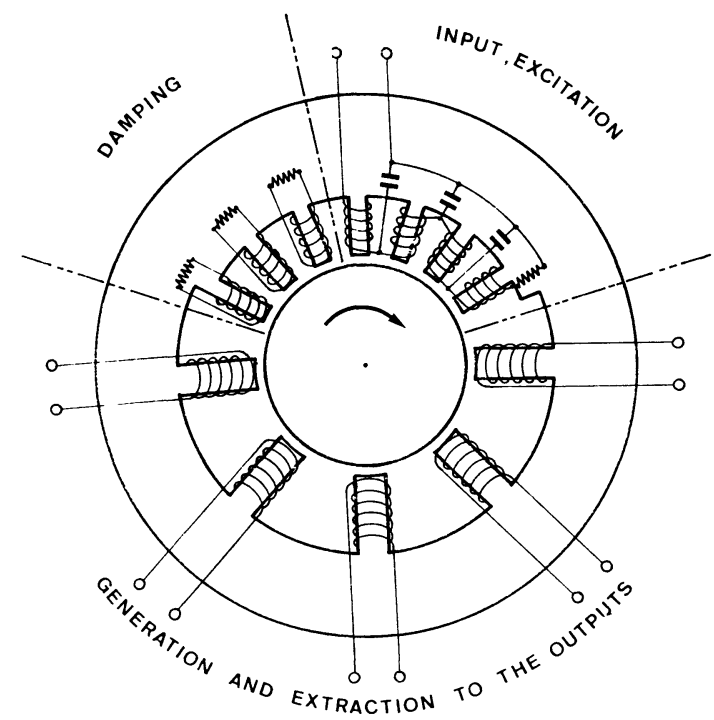

Fig. 11. - Diagram of a high power amplifier with several outputs.

Conclusion. - Starting from a comparison between microwave electron devices and rotating electrical machinery, it has been possible to evolve simple and abstract models on which one can, by field analysis, study the basic facts of the electromagnetic interaction between two structures in uniform relative motion. The basic model chosen is restrictive in several ways :

a) It is repetitive in the direction of motion, uniform in the one perpendicular direction. In fact, the spatial period is soon made infinitesimal and the system becomes uniform in the direction of motion also;

b) Only two special cases of field maps are considered ;

c) Parametric interaction is left out.
No difficulty, except complication, is expected to arise in treating all sorts of field maps. Parametric interaction is essential in few devices.

The field maps that are considered are special cases of the TE and TM modes of waveguide theory. The first one is used essentially in microwave electron devices, the second one in rotating machinery.

Aside from the field maps, the devices differ mainly by the mode of mechanically driving the moving structure, and the way energy is fed to or drawn from the two structures. These features are introduced in the theory in a natural way, often as boundary conditions.

The analysis is very close to current expositions of the theory of microwave devices, and very different from the usual text books on electrical machinery, though it permits to study with simple equations the behaviour of most machines, and to calculate their parameters in a crude way.

Its merit is to show with precision a number of similarities between all the machines considered, mainly in respect to the phase velocities and the relative velocity of the two structures forming the machine.

In Part Two of this paper, we have described some new rotating machines that can do the same things as microwave tubes : amplify a wide band of frequencies, oscillate at variable frequencies, etc...

Of course, it is difficult to evaluate their technical value ; it is a fact that some of them were patented long ago and are, to our knowledge, still not used. The main advantages of these machines would reside in their faculty of operating on rapidly varying charges without large inductive effects. Thus, the field of application might be high frequency current machines a field that can expand due to the spectacular improvements of rectifiers.

Perhaps, the advent of superconducting machinery and the increase in energy/weight factors of capacitors, plus more sophisticated demands from the users, as for instance controlled nuclear fusion engineers, will help making these machines practical and useful.

One may remark that a machine corresponding to the principle described in Part Two, paragraph 6.6 could, for the most part, be similar in structure to an asynchronous motor, even though it could operate as wideband amplifier.

It is thus apparent that some of the machines described here would differ essentially by the presence of capacitors associated with the stator; these would be fairly cumbersome except if low inductance superconductive circuits were used.

Whatever the future of the new devices may be, we think that, with some simplifications and improvements, the mode of exposition presented here for the principles of the machines is the best for students who have had a thorough course in electromagnetic theory and wave propagation phenomena.

Appendix I. - We may consider a moving frame of reference, travelling at the mean velocity of the elec- 
trons. In this frame of reference, the equations of motion may be written and integrated in the following manner, all the signals being assumed to be timesinusoidal.

$$
\begin{aligned}
\frac{\mathrm{d} \mathbf{v}}{\mathrm{d} t} & =\frac{e}{m} \mathbf{E}, \\
\mathbf{v} & =\frac{e}{m} \frac{\mathbf{E}}{j \omega} .
\end{aligned}
$$

If $\rho_{0}$ is the density of the electrons assumed to be uniform, we have :

$$
\mathbf{J}=\rho_{0} \frac{e}{m} \frac{\mathbf{E}}{j \omega}
$$

which gives, in Maxwell's equations :

$$
\operatorname{curl} \mathbf{H}=j \omega \varepsilon_{0} \mathbf{E}+\frac{\rho_{0} e}{j \omega m} \mathbf{E}
$$

introducing the total current $\mathbf{J}_{\mathbf{t}}$ :

$\mathbf{J}_{\mathbf{t}}=j \omega \varepsilon_{0} \mathbf{E}+\frac{\rho_{0} e}{j \omega m} \mathbf{E}=j \omega \varepsilon_{0} E\left(1-\frac{\rho_{0} e}{m \varepsilon_{0} \omega^{2}}\right)$.

If the map of the fields is assumed to be fixed in a moving frame of reference, we may consider a section of a tube of force having a cross section $S$ and length $l$ and use the notations:

$$
\begin{aligned}
& V=|E| l \\
& I=\left|J_{\mathbf{t}}\right| S .
\end{aligned}
$$

We will then have :

$$
\frac{I}{V}=j \omega \frac{\varepsilon_{0} S}{l}+\frac{1}{j \omega} \frac{\rho_{0} e S}{m l} .
$$

The admittance of this section of a tube of a force therefore corresponds to a capacitance $\varepsilon_{0} S / l$ connected in parallel with an inductance of value $\mathrm{ml} / \rho_{0} e S$.

Appendix II. - From equation (23), the variation of the fields in a homogeneous medium is given by the equations :

$$
\begin{aligned}
E_{z} & =A \mathrm{e}^{\gamma y}+B \mathrm{e}^{-\gamma y} & H_{z} & =C \mathrm{e}^{\gamma y}+D \mathrm{e}^{-\gamma y} \\
j \omega \mu H_{x} & =-\gamma\left[A_{\mathrm{e}}^{\gamma y}-B_{\mathrm{e}}^{-\gamma y}\right] & j \omega \varepsilon E_{x} & =\gamma\left[C \mathrm{e}^{\gamma y}-D \mathrm{e}^{-\gamma y}\right] .
\end{aligned}
$$

These equations are analogous to those of transmission lines except that the propagation constants and the characteristic impedances are imaginary : they concern waves that are evanescent in the direction $y$. As shown by equations (24) and (25), this is due to the fact that these waves are slow in the direction $x$, in order that the moving part may move at roughly their phase velocity. Only waves faster than light in the direction $x$ could be propagated at the same time in the direction $y$.

The treatment, parallel to that of transmission lines, results in the following equations :

$$
\begin{array}{rrrr} 
& E(y)=E(0) \cosh (\gamma y)-\frac{j \omega \mu H_{x}(0)}{\gamma} \sinh (\gamma y) & H(y)=H(0) \cosh (\gamma y)+\frac{j \omega \varepsilon}{\gamma} E_{x}(0) \sinh (\gamma y) \\
+\frac{j \omega \mu H_{x}(y)}{\gamma}=E(0) \sinh (\gamma y)+\frac{j \omega \mu H_{x}(0)}{\gamma} \cosh (\gamma y) & -\frac{j \omega \varepsilon E_{x}}{\gamma}=-H(0) \sinh (\gamma y)-\frac{j \omega \varepsilon}{\gamma} E_{x}(0) \cosh (\gamma y) \\
\text { or, } \quad \begin{aligned}
\gamma \\
\end{aligned} & H_{x}(y)=\frac{-\gamma \sinh (\gamma y)}{j \omega \mu} E(0)+H_{x}(0) \cosh (\gamma y) & E_{x}(y)=\frac{\gamma \sinh (\gamma y)}{j \omega \varepsilon} H(0)+E_{x}(0) \cosh (\gamma y) .
\end{array}
$$

We also obtain the transfer formulas of the impedances of the admittances :

$$
Z_{+}(y)=-\frac{j \omega \mu}{\gamma} \frac{Z(0)-\frac{j \omega \mu}{\gamma} \tanh (\gamma y)}{-\frac{j \omega \mu}{\gamma}+Z(0) \tanh (\gamma y)} \quad Y_{+}(y)=-\frac{j \omega \varepsilon}{\gamma} \frac{Y(0)-\frac{j \omega \varepsilon}{\gamma} \tanh (\gamma y)}{-\frac{j \omega \varepsilon}{\gamma}+Y(0) \tanh (\gamma y)} .
$$

\section{References}

[1] Pierce, J. R., Travelling Wave Tubes (Van Nostrand, New York) 1950.

[2] Cutler, C. C. and Mathews, W. E., Wave Transmission Amplifier U. S. Patent No 2608 623, filed June 18, 1949.

[3] Gould, R. W., Space Charge Effects in Beam-Type Magnetrons, J. Appl. Phys. 28 (1957) 599-605.

[4] Beck, A. H. W., Space Charge Waves (Pergamon Press, London) 1958.

[5] Cullen, A. L. and Barton, T. H., A simplified theory of the induction motor, using the concept of wave impedance Proc. IEE vol. 105, C 8 Sept 1958.

[6] NASAR, S. A., Electromagnetic theory of electrical machines Proc. IEE vol. 1116 June 1964.

[7] Freeman, E. M., Travelling waves in induction machines : input impedance and equivalent circuits. Proc. IEE vol. 11512 Dec. 1968.

[8] FreEman, E. M., Equivalent circuits from electromagnetic theory: low frequency induction devices. Proc. IEE vol. 12110 Oct. 1974

[9] Mourier, G., Nouvelle Machine Electrique, French Patent $\mathrm{n}^{\circ} 74$ 24667, filed on July 16, 1974, Addition $\mathrm{n}^{\circ} 75$ 33461, filed on Oct. 31st, 1975.

[10] Mourier, G., Nouvelle Machine Génératrice, Amplificatrice, French Patent $\mathrm{n}^{\circ} 75$ 34160, filed on Nov. 7th, 1975.

[11] MOURIER, G., Machine électrique tournante, amplificatrice à fréquence et amplitude rapidement variables et contrôlées, French Patent $\mathrm{n}^{\circ} 7632714$ filed on Oct. 29th, 1976. 